\title{
Altered resting state cortico-striatal connectivity in mild to moderate stage Parkinson's disease
}

\author{
Youngbin Kwak', Scott Peltier ${ }^{2}$, Nicolaas I. Bohnen ${ }^{3,4}$, Martijn L. T. M. Müller ${ }^{3}$, Praveen Dayalu ${ }^{4}$ and \\ Rachael D. Seidler ${ }^{1,5,6,7 *}$
}

1 Neuroscience Program, University of Michigan, Ann Arbor, MI, USA

2 Department of Biomedical Engineering, University of Michigan, Ann Arbor, MI, USA

${ }^{3}$ Department of Radiology, University of Michigan, Ann Arbor, MI, USA

${ }^{4}$ Department of Neurology, University of Michigan, Ann Arbor, MI, USA

${ }^{5}$ School of Kinesiology, University of Michigan, Ann Arbor, MI, USA

${ }^{6}$ Department of Psychology, University of Michigan, Ann Arbor, MI, USA

7 Institute of Gerontology, University of Michigan, Ann Arbor, MI, USA

\section{Edited by:}

Barry Horwitz, National Institutes of Health, USA

\section{Reviewed by:}

Bruno B. Averbeck, National Institute of Mental Health, USA

Michelle Hampson, Yale University, USA

\section{*Correspondence:}

Rachael D. Seidler, 401 Washtenaw Ave., Ann Arbor, MI 48109-2214, USA. e-mail: rseidler@umich.edu
Parkinson's disease (PD) is a progressive neurodegenerative disorder that is characterized by dopamine depletion in the striatum. One consistent pathophysiological hallmark of PD is an increase in spontaneous oscillatory activity in the basal ganglia thalamocortical networks. We evaluated these effects using resting state functional connectivity MRI in mild to moderate stage Parkinson's patients on and off L-DOPA and age-matched controls using six different striatal seed regions. We observed an overall increase in the strength of cortico-striatal functional connectivity in PD patients off L-DOPA compared to controls. This enhanced connectivity was down-regulated by L-DOPA as shown by an overall decrease in connectivity strength, particularly within motor cortical regions. We also performed a frequency content analysis of the BOLD signal time course extracted from the six striatal seed regions. PD off L-DOPA exhibited increased power in the frequency band $0.02-0.05 \mathrm{~Hz}$ compared to controls and to PD on L-DOPA. The L-DOPA associated decrease in the power of this frequency range modulated the L-DOPA associated decrease in connectivity strength between striatal seeds and the thalamus. In addition, the L-DOPA associated decrease in power in this frequency band correlated with the L-DOPA associated improvement in cognitive performance. Our results demonstrate that PD and L-DOPA modulate striatal resting state BOLD signal oscillations and cortico-striatal network coherence.

Keywords: dopamine, Parkinson's disease, fMRI, functional connectivity, striatum, neural oscillation

\section{INTRODUCTION}

Parkinson's disease $(\mathrm{PD})$ is a progressive neurodegenerative disorder associated with predominantly motor symptoms such as tremor, slowness of movement, rigidity, and difficulties with gait and balance, although cognitive and affective symptoms also occur (Shohamy et al., 2006; Caballol et al., 2007). The Braak staging system describes $\mathrm{PD}$ as a schema of ascending pathology, beginning in the lower brain stem and anterior olfactory structures, progressing to the basal mid- and forebrain nuclei, and then to the cortex (Braak et al., 2003, 2006). In stage 3 of the Braak system, when motor symptoms first begin to appear, the neuropathology of PD is characterized by a loss of dopaminergic neurons in the substantia nigra pars compacta and the ventral tegmental area with degeneration of the striatal nerve terminals (Braak et al., 2006).

One consistent pathophysiological hallmark of PD is an increase in spontaneous oscillatory activity in the basal ganglia thalamocortical networks (Gatev et al., 2006; Hammond et al., 2007). This increase in neural oscillatory activity is most prominent in the $10-35 \mathrm{~Hz}$ range (beta frequency band) and is often observed in local field potential recordings from the subthalamic nucleus (STN) (Kuhn et al., 2004, 2006; Foffani et al., 2005). Dopaminergic treatment suppresses this abnormally heightened oscillatory neural activity (Brown et al., 2001; Levy et al., 2002). Recently, EEG and MEG studies have shown that the increase in subcortical oscillatory activity is associated with increased intracortical coupling of neural activity, which is correlated with disease severity (Williams et al., 2002; Silberstein et al., 2005; Stoffers et al., 2008; Stam, 2010). For example, Stoffers et al. (2008) used MEG to explore how resting state cortical functional connectivity evolves over the course of PD. They found that even in recently diagnosed drug-naïve patients, there was an increase in correlations between time series in the alpha 1 frequency band measured by synchronization likelihood. Moreover, disease severity was correlated with theta and beta band synchronization (Stoffers et al., 2008). Silberstein et al. (2005) also found that EEG coherence in the $10-35 \mathrm{~Hz}$ range correlated with PD symptom severity (as measured via UPDRS). Treatments such as L-DOPA or DBS reduce this coherence, and the degree of reduction is correlated with clinical improvement (Silberstein et al., 2005).

Resting state functional connectivity MRI (fcMRI) is a noninvasive imaging technique with good spatial resolution. It identifies brain regions exhibiting correlated patterns of spontaneously occurring, slow changes in brain activity. Brain regions with similar functions and known anatomical connections have shown strong correlations in the low frequency blood oxygen level dependent 
(BOLD) signal when participants are at rest (Fox and Raichle, 2007; Rogers et al., 2007; Vincent et al., 2007). Given its non-invasive nature, it can be used to study network functional connectivity in mild to moderate stage PD patients, as opposed to the intraoperative recordings that are restricted to more advanced stage $\mathrm{PD}$ patients. Examples of functional networks that have been identified with fcMRI in healthy individuals include motor cortical networks (Biswal et al., 1995; Peltier et al., 2005), cortico-striatal networks (Di Martino et al., 2008; Kelly et al., 2009), and the default mode network (Greicius et al., 2008, 2009). Resting state connectivity networks exhibit stability across data sets collected from different participants using differing acquisition parameters, locations, and scanners (Biswal et al., 2010) making the approach well-suited for future large scale clinical studies.

A recent fcMRI study identified distinctive cognitive, motor, and reward cortico-striatal circuitries (based on connectivity between individual striatal seed regions and their cortical projection targets) in healthy young adults (Di Martino et al., 2008). A follow-up study by the same group documented that these connectivity patterns are modulated by L-DOPA administration in healthy young adults (Kelly et al., 2009). In their study, functional connectivity between the putamen and cerebellum and between the inferior ventral striatum and the ventrolateral prefrontal cortex increased with L-DOPA administration, whereas ventral striatum and dorsal caudate connectivity with the default mode network decreased. This group interpreted that L-DOPA significantly changes the motor and cognitive networks of the cortico-striatal pathways.

Cortico-striatal networks have also been examined in PD using fcMRI ( Wu et al., 2009; Helmich et al., 2010). Both studies found that compared to controls, PD patients showed increased functional connectivity in some cortico-striatal networks, and decreased connectivity in others. However, these studies only compared PD patients in the OFF medication state to controls and did not examine changes in connectivity patterns with dopaminergic medication.

In the current study, we investigated differences in cortico-striatal functional connectivity networks between $\mathrm{PD}$ patients and age matched controls, using the seed regions employed by Di Martino et al. (2008) and Kelly et al. (2009). Considering the abnormal coherence of cortico-striatal oscillatory activity reported in studies that used EEG and/or local field potential recordings (Williams et al., 2002; Kuhn et al., 2004, 2006; Foffani et al., 2005; Silberstein et al., 2005; Stoffers et al., 2008; Stam, 2010), we hypothesized that PD patients would exhibit hyperconnectivity of cortico-striatal networks in comparison to controls, and that a clinically relevant dose of L-DOPA would alleviate this hyperconnectivity. Given that PD patients exhibit increased oscillatory neural activity primarily in the alpha and beta frequency bands when off medication (Priori et al., 2004), we analyzed the frequency content of the resting state BOLD signal in the striatal seed regions. Recent work using simultaneous EEG-fMRI demonstrates that changes in the alpha and beta frequency content of neuronal activity are reflected in the BOLD signal (Goldman et al., 2002; Laufs et al., 2003; Moosmann et al., 2003; Laufs, 2008; Rosa et al., 2010). We hypothesized that frequency content of the resting state BOLD signal extracted from the seed regions would be different in PD off L-DOPA compared to controls and to PD on L-DOPA. Furthermore, we predicted that the L-DOPA associated change in the frequency content would modulate the L-DOPA associated change in cortico-striatal functional connectivity.

\section{MATERIALS AND METHODS PARTICIPANTS}

Twenty-five mild to moderate stage (Hoehn and Yahr stages 1-2.5) (Hoehn and Yahr, 1967) PD patients (64 \pm 8 years, 3 females) and 24 age- and gender-matched healthy controls ( $63 \pm 7$ years, 5 females) participated in the study. Patients were evaluated using the motor section of the Unified Parkinson's Disease Rating Scale (UPDRS) (Fahn et al., 1987) by a neurologist. The more affected body side was determined by asking each PD patient and was confirmed by the neurologist's rating. All study participants underwent the MiniMental State Exam (MMSE) (Folstein et al., 1975), the Montreal Cognitive Assessment (MOCA) (Nasreddine et al., 2005) and the grooved pegboard test (Lafayette Instruments, Lafayette, IN) to measure general cognitive and motor abilities. Performance on the grooved pegboard test has been shown to be associated with individual PD patients' dopaminergic denervation levels, as indicated by $\left[{ }^{11} \mathrm{C}\right]$ beta-CFT PET scans (Bohnen et al., 2007). These clinical assessments were acquired for patients in both the ON and OFF medication states on separate days in a counterbalanced order. The demographic and clinical characteristics of the patients are listed in Table S1 in Supplementary Material. All of our participants were diagnosed within 15 years and were in the mild to moderate stage of the disease as shown by the Hoehn and Yahr scale (Hoehn and Yahr, 1967). Participants signed a consent form approved by the Institutional Review Board of the University of Michigan prior to participation, and were compensated for their participation. All experimental procedures were conducted in accordance with the Institutional Review Board of the University of Michigan.

\section{PROCEDURE}

Parkinson's disease patients completed two testing days corresponding to the $\mathrm{ON}$ and OFF medication states. Thirteen patients were tested ON first and 12 OFF first. We used a single-blind placebo controlled design using a single dose of L-DOPA. PD patients attended both testing days in the OFF state achieved by withdrawal from medication $12-18 \mathrm{~h}$ prior to testing. For the ON testing day, patients received a $50 \mathrm{mg}$ dose of carbidopa followed by a single dose of L-DOPA in combination with carbidopa (200 mg of L-DOPA and an additional $50 \mathrm{mg}$ of carbidopa). For the OFF testing day, they received placebo medications following the same time schedule in combination with the $50 \mathrm{mg}$ of carbidopa. All study procedures began $1 \mathrm{~h}$ after the patient had taken either L-DOPA or the placebo, by which time L-DOPA reaches its peak plasma dose. Control participants underwent a single testing session without any medication procedure.

\section{fMRI DATA ACQUISITION}

fMRI data were collected from a 3 T GE Signa MRI scanner at the University of Michigan. A single-shot gradient-echo (GRE) reverse spiral pulse sequence (Glover and Law, 2001) was used to collect $240 \mathrm{~T}^{\star}$ - weighted BOLD images $(\mathrm{TR}=2 \mathrm{~s}, \mathrm{TE}=30 \mathrm{~ms}$, flip angle $=90^{\circ}, \mathrm{FOV}=220 \mathrm{~mm} \times 220 \mathrm{~mm}$, voxel size $=3.4 \mathrm{~mm}$ $\times 3.4 \mathrm{~mm} \times 3.2 \mathrm{~mm}, 40$ axial slices). For the structural images, a 
3D T1 axial overlay $\left(\mathrm{TR}=8.9 \mathrm{~ms}, \mathrm{TE}=1.8 \mathrm{~ms}\right.$, flip angle $=15^{\circ}$, $\mathrm{FOV}=260 \mathrm{~mm} \times 260 \mathrm{~mm}$, slice thickness $=1.4 \mathrm{~mm}, 124$ slices; matrix $=256 \times 160$ ) was acquired for anatomical localization. To facilitate normalization, a 110 sliced (sagittal) inversion-prepped T1-weighted anatomical image using spoiled gradient-recalled acquisition in steady state (SPGR) imaging (flip angle $=15^{\circ}$, $\mathrm{FOV}=\mathrm{FOV}=260 \mathrm{~mm} \times 260 \mathrm{~mm}, 1.4 \mathrm{~mm}$ slice thickness) was acquired. A visual fixation cross was presented to the subject using a rear projection visual display. Participants were instructed to keep their eyes centered on the cross and to not think about anything in particular. The duration of data collection was $8 \mathrm{~min}$. A pressure belt was placed around the abdomen of each subject to monitor the respiratory signal. A pulse oximeter was placed on the subject's finger to monitor the cardiac signal. The respiratory, cardiac, and fMRI data collection were synchronized.

\section{fMRI DATA ANALYSIS}

The acquired functional MRI data were preprocessed as part of the standard processing stream at the University of Michigan. First, $\mathrm{k}$-space outliers in the raw data time course greater than two standard deviations from the mean were replaced with the average of their temporal neighbors. Second, images were reconstructed using field map correction to remove distortions from magnetic field inhomogeneity. Third, physiological variations in the data from the cardiac and respiratory rhythms were removed using a regression analysis (Glover et al., 2000). This approach removed the effects of the first and second order harmonics of the externally collected physiological waveforms. Fourth, slice timing differences were then corrected using local sinc interpolation (Oppenheim et al., 1999). Finally, we used MCFLIRT in the fMRIB Software Library (Jenkinson et al., 2002) to perform motion correction (using the 10th image volume as the reference). For all participants, head motion was less than $3 \mathrm{~mm}$ in the $x, y$, or $z$ direction.

The preprocessed data were then normalized to MNI space using SPM5 (Wellcome Department of Cognitive Neurology, London, UK; http://www.fil.ion.ucl.ac.uk). We first registered the 3D T1 axial overlay to the functional images and then registered the high-resolution SPGR image to T1 overlay. The transformation to align the SPGR image to the MNI template was finally applied to the functional data. In order to have all of the PD patients' predominantly disease affected hemisphere aligned, we flipped the $x$ direction (i.e., left-right direction) of both the 240 functional images and the anatomical images for the 7 left-side more affected patients before spatial normalization. The results are presented with the left side of the images reflecting patients' more affected brain hemisphere. The same proportion of control subject images was also flipped in the $x$ direction.

The following procedures were used to generate functional connectivity images (low frequency time course correlation maps). First, the data were low-pass filtered by convolving the time courses with a rectangular filter with a cutoff frequency of $0.08 \mathrm{~Hz}$, in order to examine the frequency band of interest and to exclude higher frequency sources of noise such as heart rate and respiration (Biswal et al., 1995; Peltier et al., 2003). Second, the time course of activity was extracted from the six striatal seed regions, selected from Di Martino et al. (2008, MNI $x$ y $z$ coordinates presented and depicted in Figure $\mathbf{S 1}$ in Supplementary Material: inferior ventral striatum (VSi ( \pm ) $99-8$ ), superior ventral striatum (VSs ( \pm ) 10150 ), dorsal caudate (DC ( \pm ) 13159 ), dorsal caudal putamen (DCP $( \pm) 2813$ ), dorsal rostral putamen (DRP $( \pm) 2586$ ), and ventral rostral putamen (VRP $( \pm) 2012-3$ ). A four voxel square on the axial plane was placed around these coordinates for the seed. Third, the time course of the seed was unit normalized to remove differences in variance between subjects. Fourth, the seed region time course from the filtered data (averaged across the four voxels) was used in a correlation analysis with all other low-pass filtered voxels in the brain to form functional connectivity maps for each striatal seed region in each participant. $Z$ scores from each participant were entered into the group-level random effects analyses, which were carried out using SPM5. We first evaluated the functional connectivity maps associated with each seed region in PD OFF, PD ON, and controls separately, using a threshold of $p<0.05$ family-wise error (FWE) correction and an extent voxel threshold of 100 (Nichols and Hayasaka, 2003). At FWE $<0.05$, control group results showed significant clusters across the whole brain for all seed regions; thus, connectivity maps generated from the different seed regions were indistinguishable. Thus we report the control group results with a more stringent threshold of FWE $<0.001$. An uncorrected threshold of $p<0.001$ and extent voxel threshold of 10 was used for between group comparisons of connectivity maps. Comparisons of either PD OFF or PD ON to the control group were performed with between subjects $t$-tests, whereas comparison of PD OFF and PD ON was done using a within subjects $t$-test. We also performed an regions of interest (ROI) analysis comparing the connectivity strengths between $\mathrm{PD}$ OFF versus $\mathrm{PD} \mathrm{ON}$ in the brain regions identified from the PD OFF versus control group comparison in order to determine whether L-DOPA corrects for the elevated connectivity in these regions. ROIs were defined as the voxel clusters identified from the PD OFF versus control group comparison of connectivity maps. For example, for the inferior ventral striatum network, the whole cluster in the dorsomedial thalamus (see Figure 3 and Table S5 in Supplementary Material.) was used as an ROI and the mean connectivity strength across all voxels within this region was compared between PD OFF and PD ON using a paired $t$-test. In cases in which there were multiple ROIs associated with one seed region (i.e., dorsal caudal putamen and dorsal rostral putamen), repeated measures ANOVA using ROI and medication status as within subject factors was performed.

We also combined the connectivity maps associated with the three caudate seed regions (inferior ventral striatum, superior ventral striatum, and dorsal caudate) and the three putamen seed regions (dorsal caudal putamen, dorsal rostral putamen, and ventral rostral putamen) using the ImCalc applet in SPM5 to perform numerical addition of the three connectivity maps (Di Martino et al. 2008). Direct comparison of the caudate and putamen connectivity maps were performed in controls, PD OFF and PD ON separately using an uncorrected threshold of $p<0.001$ and extent voxel threshold of 10 .

\section{FREOUENCY CONTENT ANALYSIS OF THE FMRI BOLD SIGNAL}

The extracted fMRI BOLD timecourses from the six striatal seeds were transformed into the frequency domain using Fast Fourier Transformation in Matlab. We low pass filtered our data 
at $0.08 \mathrm{~Hz}$, and thus we were only able to look at the power spectrum between 0.0 and $0.08 \mathrm{~Hz}$. For the normalization of the frequency data we took the following steps. We first divided the $0.08 \mathrm{~Hz}$ spectrum into eight frequency bands of approximately $0.01 \mathrm{~Hz}$ bandwidth each. We then computed the percentage of the total power contained within each frequency band. Repeated measures ANOVA was used to compare the normalized power content across the eight frequency bands for PD ON and OFF medication, and a mixed model ANOVA was used for the comparison of the PD patients to the controls using frequency bands as a within subject factor and group (PD OFF versus controls) as a between subject factor. The Huynh-Feldt epsilon (Huynh and Feldt, 1970) was used to determine whether the repeated measures data met the assumption of sphericity $(\Sigma>0.75)$. In cases where the sphericity assumption was not met, the $F$ statistic was evaluated for significance using the Huynh-Feldt adjusted degrees of freedom.

\section{RESULTS \\ BEHAVIORAL DATA}

We evaluated patients' performance on the UPDRS, MMSE, MOCA, and grooved pegboard tests between the ON and OFF L-DOPA states using paired $t$-tests. Pegboard performance was analyzed separately for the more and less affected sides. Motor symptoms measured by UPDRS were significantly worse in PD OFF than PD ON $\left(t_{24}=-2.33, p<0.05\right)$, and pegboard performance for the more affected side was significantly worse for PD OFF than PD ON $\left(t_{24}=-2.88, p<0.01\right)$. These results indicate that L-DOPA significantly improved motor functioning of the patients, and the improvement was most apparent in the more affected side.

\section{STRIATAL FUNCTIONAL CONNECTIVITY IN CONTROLS, PD OFF, AND PD ON}

We present our connectivity analyses using the seeds placed in the more affected hemisphere; results were generally similar in the less affected hemisphere. We first identified striatal functional connectivity maps for the control group to evaluate the anatomical plausibility of the networks (FWE correction of $<0.001$ ). In general, the results paralleled previous findings (Di Martino et al., 2008; Kelly et al., 2009) (Figure 1). Networks for the three caudate seeds followed the ventral-dorsal distinction of cortico-striatal connectivity, such that the inferior ventral striatum showed connectivity with the ventral medial prefrontal areas and anterior cingulate cortex while the superior ventral striatum and dorsal caudate showed connectivity with the more dorsal and lateral areas of the prefrontal cortex including the dorsolateral prefrontal cortex (BA 46) (Table S2 in Supplementary Material). The three putamen seeds showed connectivity with the primary and secondary motor cortical areas as well as prefrontal and parietal association cortical regions (Table S2 in Supplementary Material). Our data also showed that there was less specificity of corticostriatal connectivity across the six striatal seed regions compared to previous studies (Di Martino et al., 2008). For example, we found motor cortical areas associated with the caudate seeds and significant involvement of prefrontal areas associated with the three putamen seeds. This may be due to the effect of agerelated decreases in the specificity of functional networks in older adults, since our control group mean age was 63 years (cf. Park and Reuter-Lorenz, 2009; Seidler et al., 2010). This remains to be evaluated in future studies.

We found relatively similar cortico-striatal connectivity patterns in each of our patient groups (Figure S2 in Supplementary Material, Tables S3 and S4 in Supplementary Material). The decreased specificity of the functional connectivity patterns of the six striatal seed regions was also present in the patient groups. In order to determine the distinctiveness of the cognitive and motor networks we directly compared the connectivity maps for caudate and putamen seeds in each group. In the control group, the regions showing greater correlations with the caudate seeds than the putamen seeds included the ventromedial prefrontal cortex, posterior cingulate and the parahippocampal gyrus (Figure 2, Table 1). The regions showing greater correlation with the putamen seeds than the caudate seeds included the primary and supplementary motor areas (Figure 2, Table 1). In PD OFF and PD ON however we did not find any regions other than the caudate and putamen themselves to be more correlated with caudate or putamen seed regions, with the exception of the dorsal prefrontal cortex (BA 8) in PD OFF and

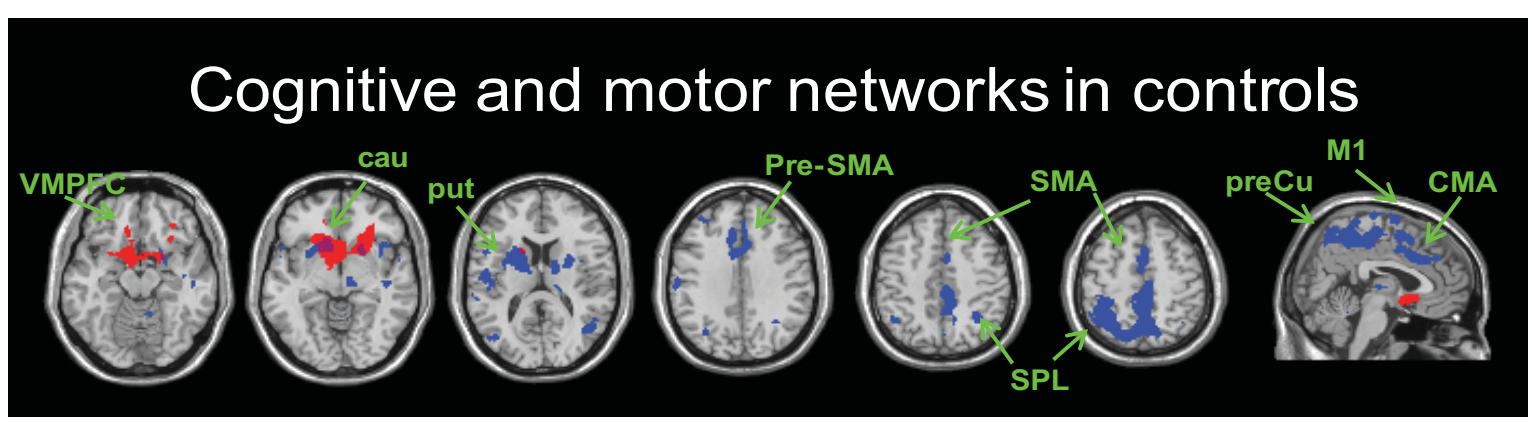

FIGURE 1 |An example of cognitive (seed: inferior ventral striatum, red) and motor (seed: dorsal rostral putamen, blue) networks in controls. $z=-15,-5$, 15, 35, 45, 55 and $x=5$ from left to right (FWE-corrected $p<0.001)$. VMPFC, ventromedial prefrontal cortex; cau, caudate; put, putamen; Pre-SMA, pre-supplementary motor area; SMA, supplementary motor area; SPL, superior parietal lobule; M1, primary motor area; CMA, cingulate motor area; preCu, precuneus. 


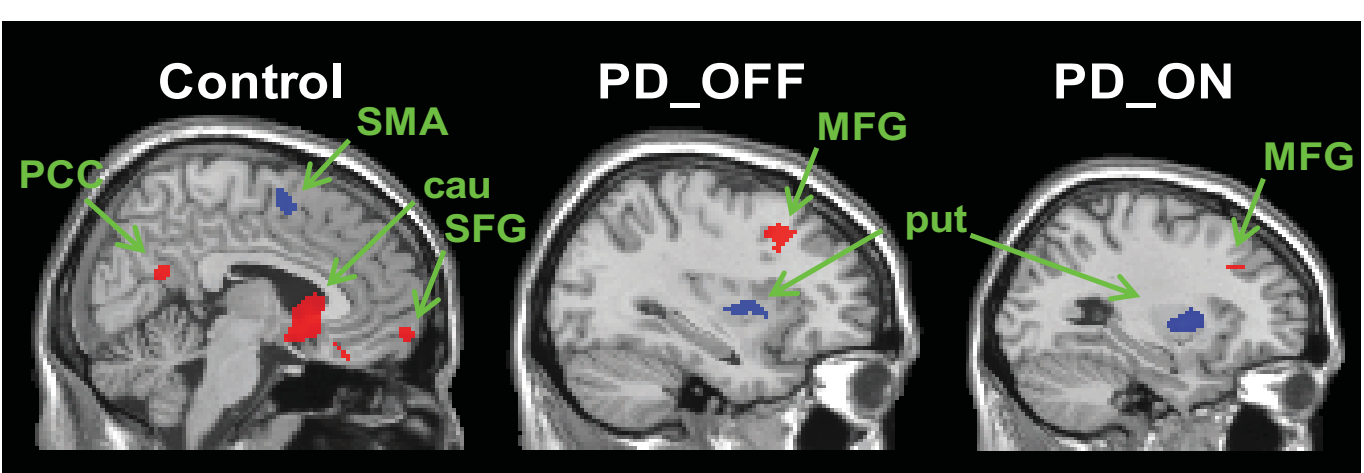

FIGURE 2 | Regions showing greater connectivity with caudate seeds (VSi, VSs, and DC combined) than with putamen seeds (DCP, DRP, and VRP

seeds than with caudate seeds in blue $(\boldsymbol{p}<\mathbf{0 . 0 0 1}$, uncorrected). PCC combined) in red and regions showing greater connectivity with putamen posterior cingulate cortex; SMA, supplementary motor area; SFG, superior frontal gyrus; MFG, middle frontal gyrus; cau, caudate; put, putamen.

Table 1 | Direct comparisons between caudate seeds combined and putamen seeds combined.

\begin{tabular}{|c|c|c|c|c|c|c|}
\hline \multirow[t]{2}{*}{ Contrast } & \multirow[t]{2}{*}{ Region } & \multirow[t]{2}{*}{ BA } & \multicolumn{3}{|c|}{ MNI coordinates } & \multirow[t]{2}{*}{$T$-value } \\
\hline & & & $\boldsymbol{x}$ & $y$ & $z$ & \\
\hline \multicolumn{7}{|l|}{ CONTROL } \\
\hline \multirow[t]{8}{*}{ Caudate $>$ putamen } & Caudate & & -12 & 14 & 8 & 264.45 \\
\hline & Inferior temporal gyrus & 20 & -68 & -20 & -14 & 6.13 \\
\hline & Parahippocamal gyrus & 36 & -26 & -18 & -22 & 5.75 \\
\hline & Middle frontal gyrus & 11 & 34 & 38 & -14 & 4.92 \\
\hline & Rectal gyrus & 11 & 8 & 32 & -18 & 4.14 \\
\hline & Superior frontal gyrus & 10 & -28 & 66 & 14 & 4.48 \\
\hline & Posterior cingulate gyrus & 23 & 6 & -58 & 20 & 4.19 \\
\hline & Inferior frontal gyrus & 47 & -52 & 32 & -12 & 3.96 \\
\hline \multirow[t]{10}{*}{ Putamen > caudate } & Putamen & & -28 & 0 & 2 & 276.94 \\
\hline & Precentral gyrus & 6 & 46 & 12 & 6 & 6.02 \\
\hline & Medial frontal gyrus (pre-SMA) & 6 & 6 & 4 & 56 & 4.32 \\
\hline & Superior frontal gyrus & 9 & 32 & 50 & 34 & 5 \\
\hline & Precuneus & 7 & 18 & -58 & 46 & 4.59 \\
\hline & Postcentral gyrus & 7 & 28 & -54 & 70 & 3.9 \\
\hline & Superior temporal gyrus & 22 & 66 & -8 & 6 & 4.64 \\
\hline & Inferior frontal gyrus & 47 & 54 & 36 & -2 & 4.25 \\
\hline & Inferior parietal lobule & 40 & -56 & -38 & 46 & 3.87 \\
\hline & Ventral posterior medial thalamus & & 18 & -22 & 4 & 3.75 \\
\hline \multicolumn{7}{|l|}{ PD OFF } \\
\hline \multirow[t]{2}{*}{ Caudate $>$ putamen } & Caudate & & 6 & 12 & 12 & 4.61 \\
\hline & Middle frontal gyrus & 8 & -30 & 10 & 40 & 4.23 \\
\hline Putamen > caudate & Putamen & & -26 & -2 & -2 & 4.33 \\
\hline \multicolumn{7}{|l|}{ PD ON } \\
\hline \multirow[t]{2}{*}{ Caudate $>$ putamen } & Caudate & & -6 & 20 & 4 & $\operatorname{lnf}$ \\
\hline & Middle frontal gyrus & 9 & -28 & 26 & 34 & 3.66 \\
\hline Putamen > caudate & Putamen & & -22 & 6 & -6 & 4.33 \\
\hline
\end{tabular}

Inf, infinite T-value.

the dorsolateral prefrontal cortex (BA 9) in PD ON which exhibited greater correlated activity with the caudate than the putamen (Figure 2, Table 1). These results indicate that in both PD OFF and PD ON there is decreased specificity of the caudate and putamen functional connectivity patterns.
COMPARISON OF CAUDATE VERSUS PUTAMEN FUNCTIONAL CONNECTIVITY IN CONTROLS, PD OFF, AND PD ON

Next, we compared the connectivity maps associated with each seed region between groups. We first compared PD OFF to controls. We found increased functional connectivity in PD OFF compared 


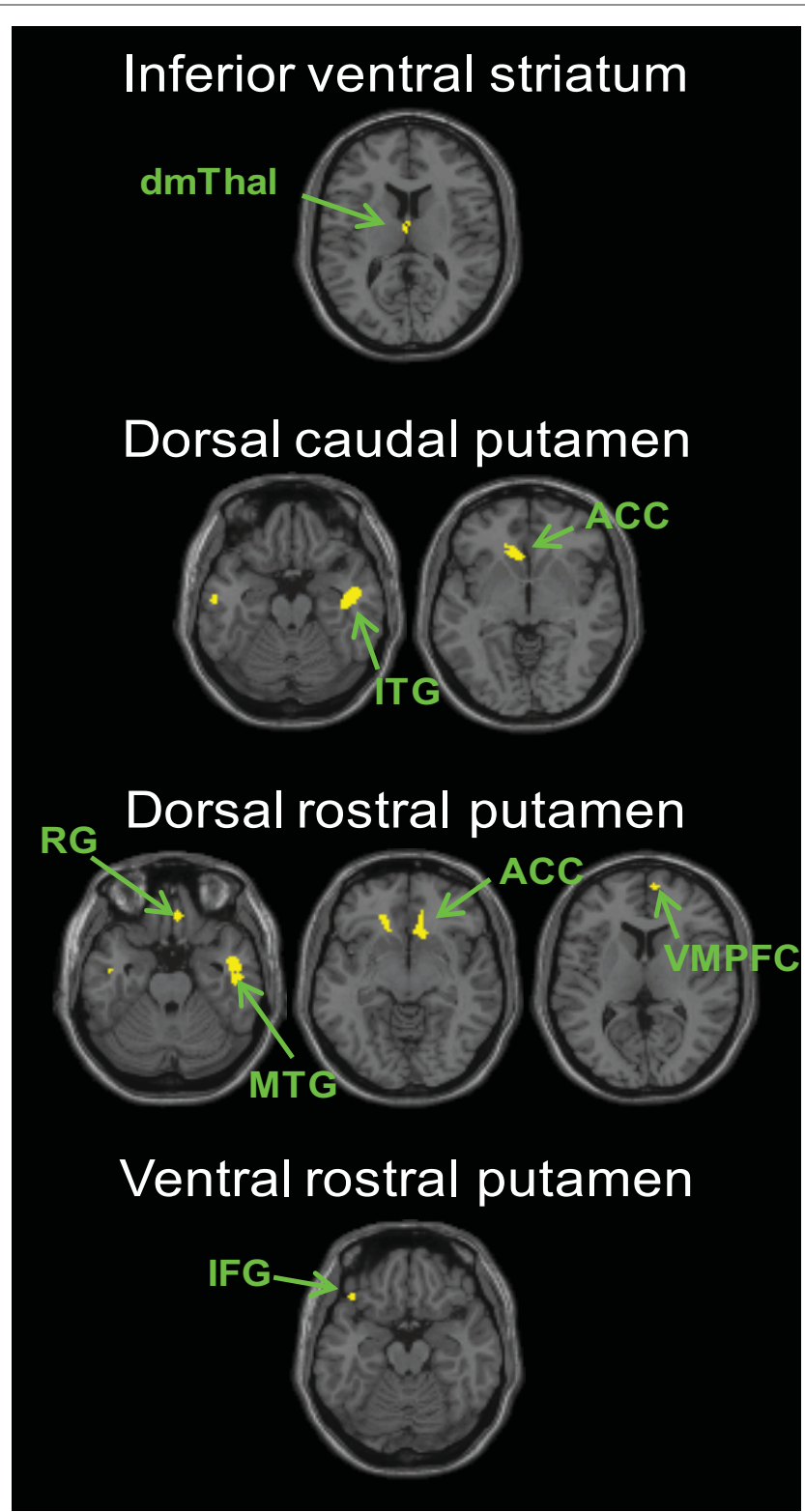

FIGURE 3 | Regions showing greater connectivity with the striatal seeds for PD OFF than controls ( $\boldsymbol{p}<\mathbf{0 . 0 0 1}$, uncorrected). No results were found in the other seeds or for the contrast of controls greater than PD OFF. dmThal, dorsomedial thalamus; ACC, anterior cingulate cortex; VMPFC, ventromedial prefrontal cortex; IFG, inferior frontal gyrus; RG, rectal gyrus; MTG, middle temporal gyrus.

to controls with the following seed regions: inferior ventral striatum, ventral rostral putamen, dorsal caudal putamen, and dorsal rostral putamen (Figure 3, Table S5 in Supplementary Material). Regarding the first two seeds, connectivity between the inferior ventral striatum and the dorsomedial thalamus and connectivity between the ventral rostral putamen and the ventromedial prefrontal gyrus was increased in PD OFF compared to controls. For dorsal caudal putamen, the inferior temporal gyrus, anterior cingulate cortex and superior frontal gyrus showed greater connectivity in PD OFF than controls. For dorsal rostral putamen, the ventrome- dial prefrontal cortex, the anterior cingulate, and the middle and inferior temporal gyrus showed greater connectivity in PD OFF than controls. The increased connectivity in PD OFF was most significantly found in the two dorsal putamen seeds, and we did not find any regions showing greater connectivity for the caudate seeds except for inferior ventral striatum. There were no regions that showed greater connectivity in controls than PD OFF. These results indicate that $\mathrm{PD}$ patients show greater cortico-striatal functional connectivity compared to controls specifically in the dorsal putamen seeds. It is of note that the denervation of dopamine in $\mathrm{PD}$ is most prominent in the dorsal and posterior striatum, including the dorsal putamen, which is selectively affected in the earlier stages of the disease (Braak et al., 2003, 2006). Considering that the PD patients in the current study were all in the relatively early stages of the disease, our results indicate that increases in functional connectivity are more prominent in the early disease affected striatal subregions.

We also compared PD ON to controls. In general we found decreased cortico-striatal functional connectivity in PD ON compared to controls (Figure 4, Table S6 in Supplementary Material). Decreases in connectivity were found in the primary and supplementary motor areas and the associative prefrontal and parietal regions irrespective of seed location. No regions exhibited greater connectivity for any of the striatal seeds in PD ON than controls.

Lastly, we compared connectivity maps for PD OFF versus PD ON. We found increased connectivity of the striatal seeds in PD OFF compared to PD ON, and the brain regions showing increased connectivity with each seed were part of the functional network associated with that particular seed (Figure 5, Table S7 in Supplementary Material). That is, for the caudate seeds, increased connectivity in PD OFF compared to PD ON was found in the ventromedial prefrontal (BA 11) and orbitofrontal (BA 10) regions for inferior ventral striatum, dorsolateral prefrontal (BA 46) and frontal eye field (BA 8) regions for superior ventral striatum and dorsal caudate. For the putamen seeds, increased connectivity in PD OFF compared to PD on was observed in the primary and secondary motor areas (BA 4, BA 6). There were no regions that showed greater connectivity in PD ON than PD OFF. These results indicate that L-DOPA decreases the abnormally high functional connectivity in $\mathrm{PD}$, with specific effects on the functional networks associated with each cortico-striatal seed. However, our findings indicate that L-DOPA reduces cortico-striatal connectivity in PD to a greater extent than is necessary as evidenced by overall decreased connectivity in PD ON compared to controls.

The brain regions identified in the voxel-wise comparison of connectivity maps between PD OFF and PD ON did not necessarily overlap with the regions identified by the PD OFF versus control group comparison. That is, from the voxel-wise comparison results we could not determine whether L-DOPA corrected the aberrantly elevated functional connectivity in PD. In order to address this, we performed an ROI analysis comparing the connectivity strengths between PD OFF and PD ON in the brain regions identified from the PD OFF versus control group comparison. The mean connectivity strengths across all voxels within the ROIs were compared between PD OFF and PD ON. For the inferior ventral striatum seed region, we found that mean connectivity strength with the dorsomedial thalamus was significantly lower in PD ON than PD OFF $\left(t_{24}=2.44, p<0.05\right.$, Figure S3A in Supplementary 


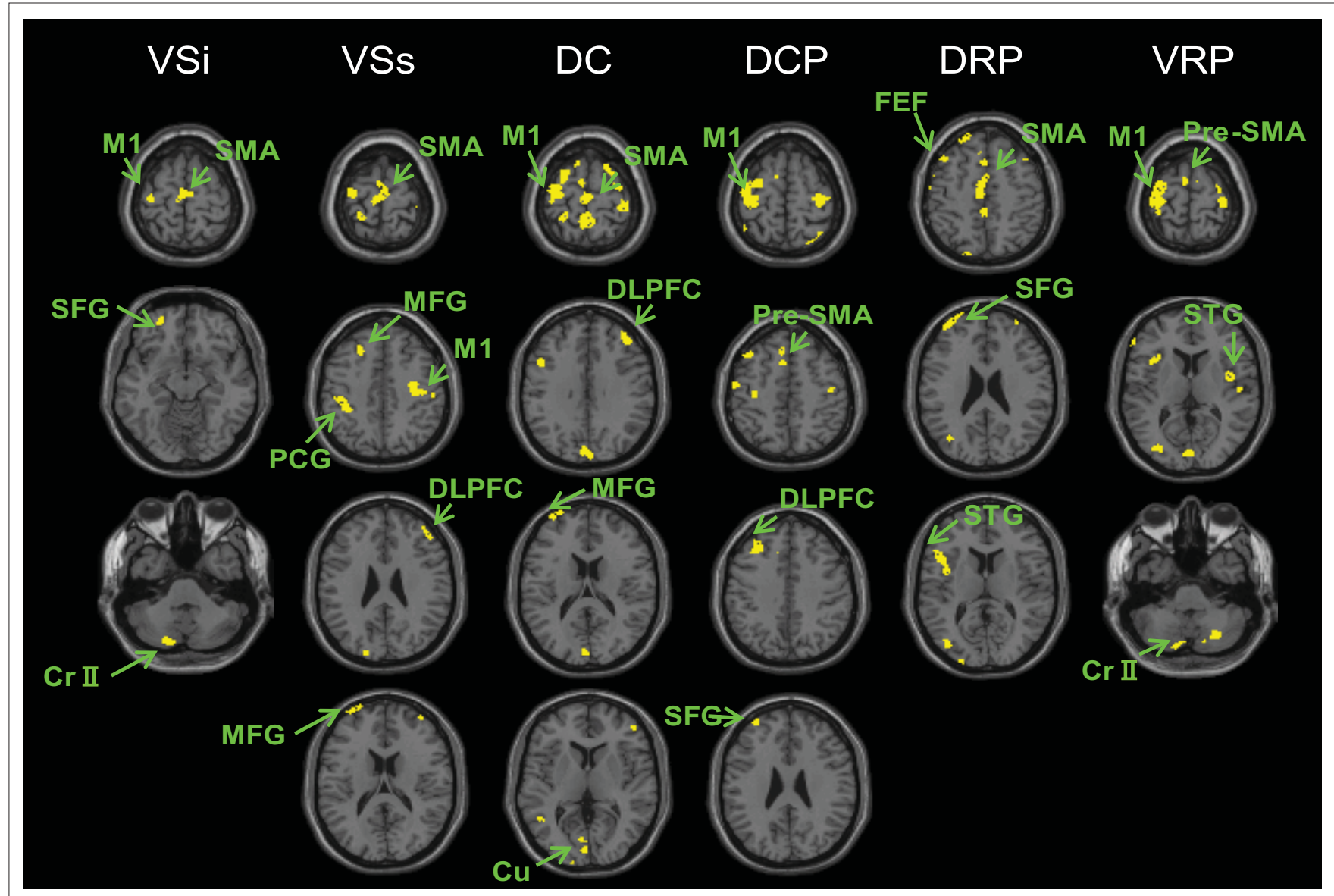

FIGURE 4 | Regions showing greater connectivity with the striatal seeds in controls than PD ON ( $\boldsymbol{p}<\mathbf{0 . 0 0 1}$, uncorrected). No results were found for the contrast of PD ON greater than controls. VSi, inferior ventral striatum; VSs, superior ventral striatum; DC, dorsal caudate; DCP, dorsal caudal putamen; DRP, dorsal rostral putamen; VRP, ventral rostral putamen; M1, primary motor cortex; SMA, supplementary motor area; pre-SMA, presupplementary motor area; DLPFC, dorsolateral prefrontal cortex; $\mathrm{Cr}$ II, cerebellum Crus II, STG, superior temporal gyrus; SFG, superior frontal gyrus; FEF, frontal eye field; cu, cuneus; MFG, middle frontal gyrus; PCG, postcentral gyrus.
Material). For dorsal caudal putamen, which had multiple ROIs associated with it, repeated measures ANOVA showed a significant main effect of medication status $\left(F_{1,24}=9.22, p<0.01\right.$, Figure S3B in Supplementary Material), reflecting an overall decrease in connectivity strength in PD ON compared to PD OFF. For dorsal rostral putamen we found a marginally significant main effect of medication status $\left(F_{1,24}=3.66, p=0.068\right.$, Figure S3C in Supplementary Material), reflecting an overall decrease in connectivity strength in PD ON compared to PD OFF. For ventral rostral putamen, connectivity strength with inferior frontal gyrus was lower in PD ON than PD OFF but was only marginally significant $\left(t_{24}=1.81\right.$, $p=0.08$, Figure S3D in Supplementary Material). These results demonstrate that L-DOPA indeed corrects for the elevated connectivity in the brain regions that show increased connectivity for PD OFF compared to controls.

\section{FREOUENCY CONTENT ANALYSIS}

Comparison of connectivity maps between groups showed that there was an overall elevation of cortico-striatal functional connectivity in PD and that L-DOPA decreased this heightened connectivity. In order to determine whether increased connectivity in PD is associ- ated with a change in oscillatory activity of the striatal BOLD signal in the resting state, we performed a frequency content analysis of the BOLD signal time course extracted from the six striatal seed regions. We first compared PD OFF to controls using a mixed between and within subjects ANOVA with group as a between subjects factor and frequency bands as a within subjects factor in each seed separately. Since we analyzed the normalized power (total power of the eight frequency bands being 100\%) there was inherently no main effect of group. We found significant group by frequency band interactions for the inferior ventral striatum $\left(F_{4.49,210.84}=2.39, p<0.05\right)$, dorsal caudate $\left(F_{7,329}=2.01, p=0.05\right)$ and dorsal caudal putamen $\left(F_{7,329}=2.46, p<0.05\right)$. We followed these up with two sample $t$-tests comparing the frequency content of PD OFF and controls in each frequency band for these seed regions. We found significant differences in the frequency content in $0.02-0.03 \mathrm{~Hz}$ for inferior ventral striatum $\left(t_{47}=2.45, p<0.05\right)$ and dorsal caudate $\left(t_{47}=2.11\right.$, $p<0.05)$, and in $0.03-0.04 \mathrm{~Hz}$ for dorsal caudal putamen $\left(t_{47}=2.27\right.$, $p<0.05)$. Normalized mean signal power was significantly greater in PD OFF than controls in these frequency ranges (Figure 6). A concomitant decrease in signal power for PD OFF was seen in the range of $0-0.02 \mathrm{~Hz}$, although it did not reach significance. 


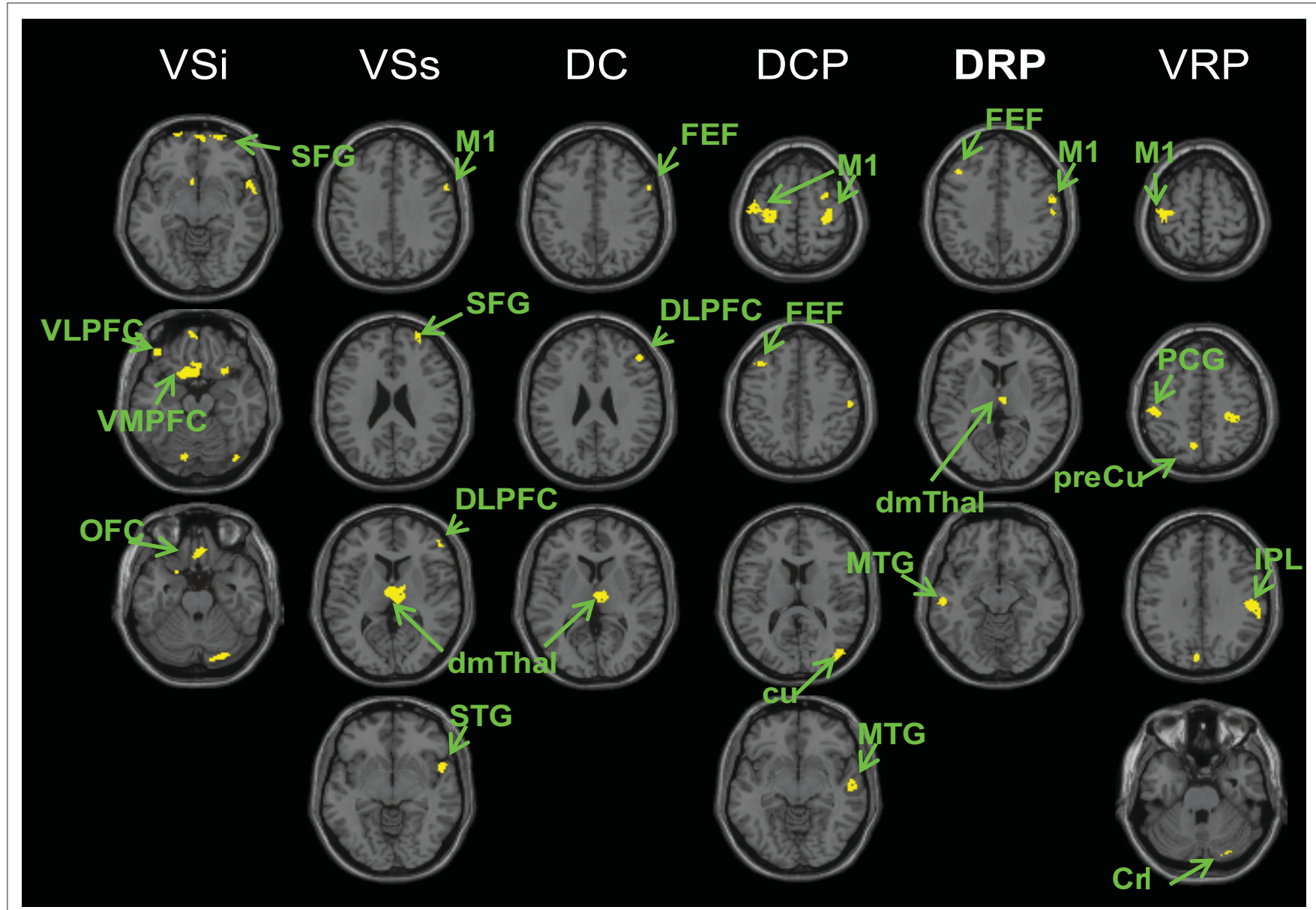

FIGURE 5 | Regions showing greater connectivity with the striatal seeds in PD OFF than PD ON ( $\boldsymbol{p}<\mathbf{0 . 0 0 1}$, uncorrected). No results were found for the contrast of PD ON greater than PD OFF. VSi, inferior ventral striatum; VSs, superior ventral striatum; DC, dorsal caudate; DCP, dorsal caudal putamen; DRP, dorsal rostral putamen; VRP, ventral rostral putamen; OFC, orbital frontal cortex;
VLPFC, ventrolateral prefrontal cortex; VMPFC, ventromedial prefrontal cortex; DLPFC, dorsolateral prefrontal cortex; FEF, frontal eye field; M1, primary motor cortex; SFG, superior frontal gyrus; STG superior temporal gyrus; dmThal, dorsomedial thalamus; MTG, middle temporal gyrus; cu, cuneus; $\mathrm{Cr}$ I, cerebellum crus I; IPL, inferior parietal lobule; PCG, post central gyrus; preCu, precuneus.
We also compared the power content for PD OFF versus PD $\mathrm{ON}$ across the eight frequency bands for each striatal seed region using repeated measures ANOVA. We found significant medication by frequency band interactions for superior ventral striatum $\left(F_{7,168}=3.53, p<0.005\right)$, dorsal caudate $\left(F_{3.97,95.18}=4.42, p<0.005\right)$, dorsal caudal putamen $\left(F_{4.34,104.15}=2.73, p<0.05\right)$ and a marginally significant interaction for dorsal rostral putamen $\left(F_{5.12,122.93}=2.2\right.$, $p=0.057$ ) (Figure 7). We followed up with paired $t$-tests comparing the frequency content of the signals for PD OFF versus PD $\mathrm{ON}$ in each frequency band for these seed regions. The results showed that across the four seeds, there was a significant difference between PD OFF and PD ON in the frequency range $0-0.02 \mathrm{~Hz}$ and $0.03-0.05 \mathrm{~Hz}$. In general, signal power in $0.03-0.05 \mathrm{~Hz}$ was greater in PD OFF than PD ON with a concomitant decrease in power in the $0-0.02 \mathrm{~Hz}$ range (VSs: $0-0.01 \mathrm{~Hz}, t_{24}=-3, p<0.01$; DC: $0-0.01 \mathrm{~Hz}, t_{24}=-2.44, p<0.05,0.01-0.02 \mathrm{~Hz}, t_{24}=-2.75$, $p<0.05,0.04-0.05 \mathrm{~Hz}, t_{24}=2.62, p<0.05$; DCP: $0-0.01 \mathrm{~Hz}$, $t_{24}=-2.03, p=0.053,0.03-0.04 \mathrm{~Hz}, t_{24}=2.48, p<0.05$; DRP: $\left.0.03-0.04 \mathrm{~Hz}, t_{24}=2.58, p<0.05\right)$. Taken together with the results arising from the comparison of PD OFF to controls, these data demonstrate that there is relatively greater power for the resting state BOLD signal oscillations in the $0.02-0.05 \mathrm{~Hz}$ frequency band for PD OFF, and L-DOPA decreases the elevated oscillations in this frequency range.

In order to determine whether this L-DOPA-associated change in power content within these specific frequency bands modulates L-DOPA-associated connectivity differences in any brain regions, we performed an exploratory voxel-wise correlation analysis. In this analysis, we performed a voxel-wise regression using the OFF and ON difference in the normalized signal content and the OFF and ON connectivity difference. Considering that both the comparisons of PD OFF to controls and PD OFF to PD ON showed significantly increased power in the $0.02-0.05 \mathrm{~Hz}$ range, we used the OFF-ON normalized signal content of the frequency bands that showed significantly greater power for PD OFF than PD ON (i.e., $0.04-0.05 \mathrm{~Hz}$ for dorsal caudate, $0.03-0.04 \mathrm{~Hz}$ for dorsal caudal putamen and dorsal rostral putamen) and the OFF-ON connectivity maps. Analyses were performed within brain regions 

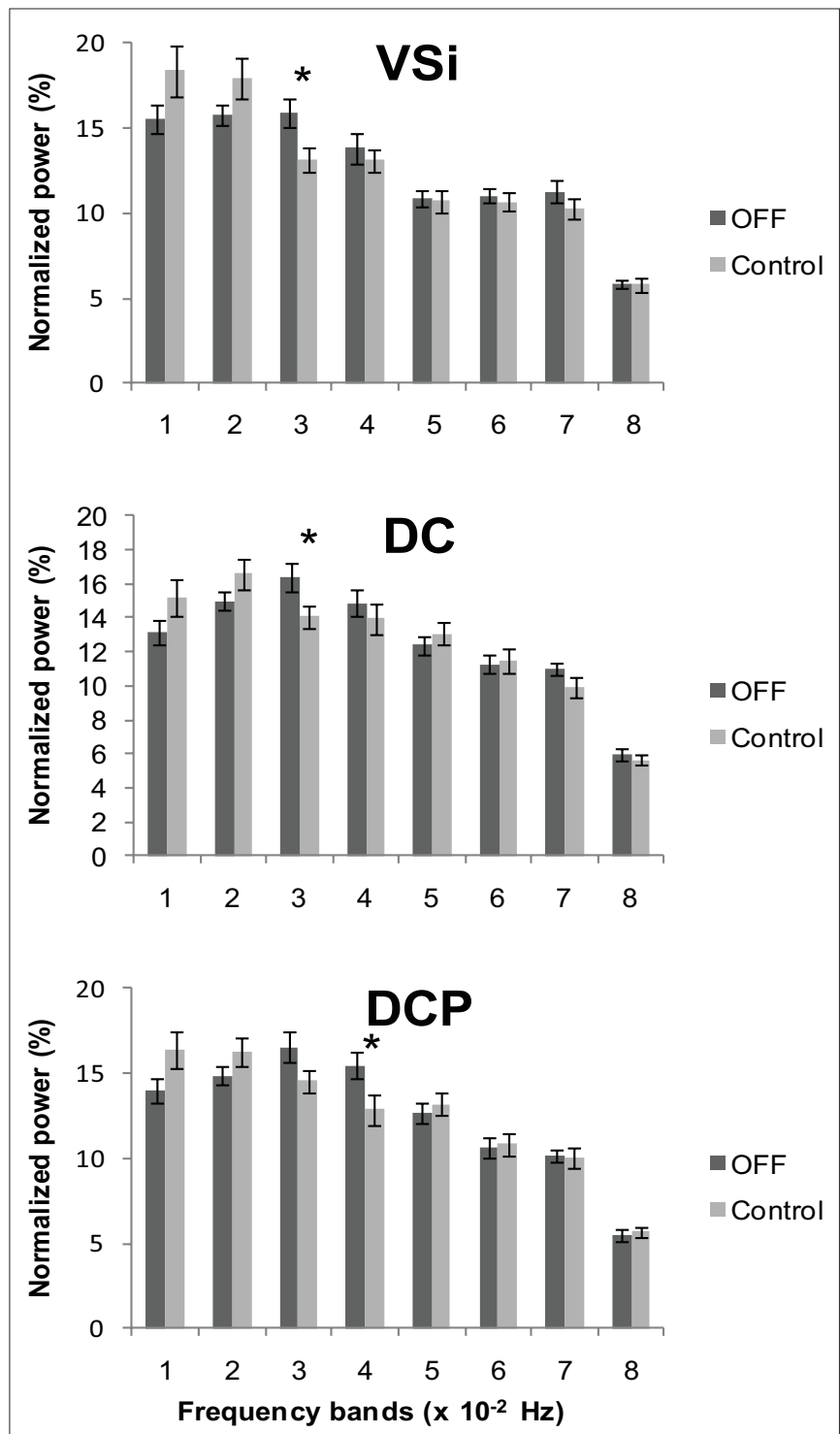

FIGURE 6 | Normalized power across the eight frequency bands in controls and PD OFF. Each band consists of approximately $0.01 \mathrm{~Hz}$ bandwidth. A significant group by frequency band interaction was found in inferior ventral striatum (VSi), dorsal caudate (DC), and dorsal caudal putamen (DCP) with PD OFF showing greater signal content in the $0.02-0.04 \mathrm{~Hz}$ range of the frequency band than controls. Error bars indicate standard error. ${ }^{*} p<0.05$.

that showed greater OFF than ON functional connectivity using an explicit mask of the OFF-ON connectivity map thresholded at $p<0.05$, uncorrected. The correlation analyses within these masks were thresholded at $p<0.005$ uncorrected with a voxel extent threshold of 10 . The results showed that for dorsal caudate, the OFF-ON frequency content difference in $0.04-0.05 \mathrm{~Hz}$ was positively correlated with OFF-ON connectivity differences within the dorsomedial thalamus. That is, greater L-DOPA-associated decreases in power in this frequency band were associated with greater decreases in connectivity strength between the dorsal caudate and the dorsomedial thalamus (Figure 8). A similar result was found for dorsal caudal putamen, which exhibited a significant positive correlation between the OFF-ON frequency content difference in $0.03-0.04 \mathrm{~Hz}$ and OFF-ON connectivity with the dorsomedial thalamus (Figure 9).

We also performed a correlation analysis between the OFF and ON difference in frequency content of the $0.03-0.05 \mathrm{~Hz}$ and the OFF and $\mathrm{ON}$ difference in behavioral measures to determine whether the change in frequency content is correlated with the medication-associated changes in behavior. We used OFF-ON frequency content and OFF-ON behavioral performance measured with MOCA, grooved pegboard, and UPDRS. We only found a significant negative correlation between OFF-ON frequency content of $0.04-0.05 \mathrm{~Hz}$ in dorsal caudate with the OFF-ON difference in MOCA score $(r=-0.44$, $p<0.05$ ) (Figure 10). That is, the greater the L-DOPA-associated decrease in power in this frequency band of dorsal caudate, the greater the improvement in MOCA performance.

Collectively, the results from our frequency content analyses of the resting state BOLD time course in the six striatal seed regions indicate that there is a relative increase in BOLD signal oscillations in the $0.02-0.05 \mathrm{~Hz}$ range in $\mathrm{PD}$, and $\mathrm{L}$-DOPA mitigates this effect. The L-DOPA-associated decrease in the frequency content in this range modulated the change in connectivity strength between dorsal caudate and thalamus and dorsal caudal putamen and thalamus. Moreover, for dorsal caudate, the change in frequency content due to medication also modulated the L-DOPA associated change in cognitive performance.

\section{DISCUSSION}

We observed hyperconnectivity of the cortico-striatal networks in PD patients, which was mitigated by L-DOPA. Comparisons of connectivity maps between PD OFF and PD ON showed decreased connectivity with L-DOPA in brain regions that are known to be anatomically connected to each of the seed regions. Additional ROI analyses comparing connectivity strength between PD OFF and PD ON within the regions that showed hyperconnectivity in PD OFF compared to controls also revealed a general decrease in connectivity strength with L-DOPA. It is of note however, that down-regulation of connectivity strength in these ROIs was not observed across the board. Therefore, although L-DOPA normalized the PD-associated hyperconnectvity it was more effective in some ROIs than others. Hyperconnectivity in PD patients is explained by the shift in the frequency content of the resting state striatal BOLD signal oscillations in PD patients. More specifically, we found that PD patients OFF L-DOPA had increased functional connectivity between striatal seed regions and cortical areas compared to controls in the two dorsal putamen seeds, which are known to be the most disease-affected subregions of the striatum in the early disease state (Bernheimer et al., 1973; Kish et al., 1988; Frey et al., 1996; Rakshi et al., 1999; Braak et al., 2003, 2006). This hyperconnectivity for PD OFF was also seen in a whole-brain comparison relative to PD ON. Furthermore, the regions showing greater functional connectivity for PD OFF than ON reflected the specificity of the reward, cognitive and motor circuitry of the cortico-striatal pathways. For example, the regions showing greater connectivity with the inferior ventral striatum in PD OFF than PD ON were structures typically involved in reward processing such as the orbital frontal and ventromedial prefrontal cortex (Haber and Knutson, 2010). The regions showing greater connectivity with the dorsal 

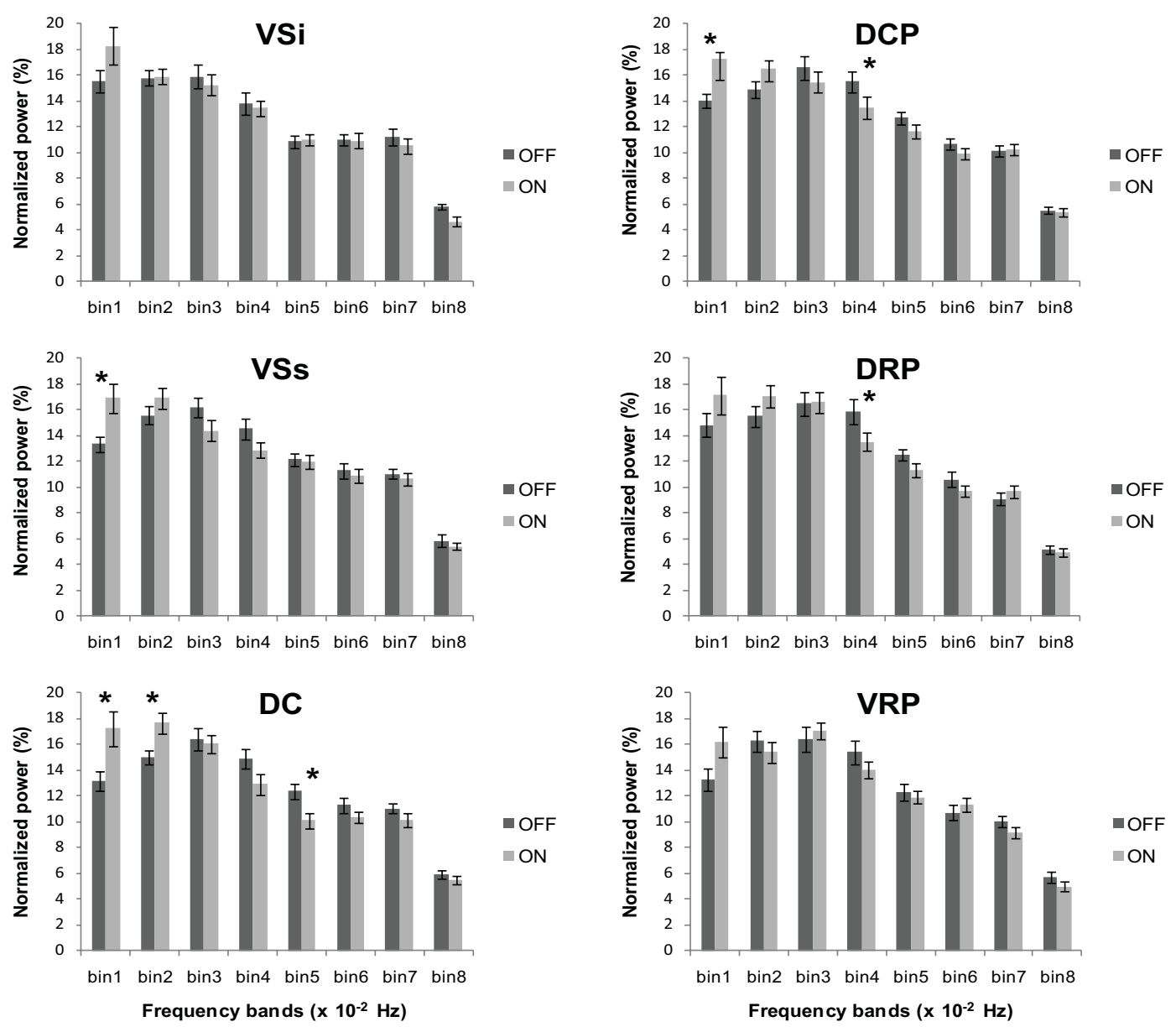

FIGURE 7 | Normalized power across the eight frequency bands in PD ON and PD OFF. Significant group by frequency band interactions were observed for the superior ventral striatum (VSs), dorsal caudate (DC), dorsal caudal putamen (DCP) and dorsal rostral putamen (DRP). Error bars indicate standard error. ${ }^{*} p<0.05$.

caudate in OFF than $\mathrm{ON}$ included the dorsolateral prefrontal cortex which is part of the cognitive cortico-striatal loop (Alexander et al., 1986), whereas for the putamen seeds, differences in connectivity strength were found with the primary and secondary motor cortical areas. These results indicate that there is an increase in the level of connectivity between striatal regions and their selective cortical targets in PD patients.

The increased connectivity we found in PD patients compared to controls is somewhat unique to this clinical group since other studies have widely shown disrupted connectivity in patients with autism, depression, schizophrenia, and stroke (Greicius, 2008; Monk et al., 2009; van Meer et al., 2010; Vercammen et al., 2010). However, our observation of hyperconnectivity in PD patients OFF medication and down-regulation of this hyperconnectivity by administration of L-DOPA, is in alignment with previous studies reporting increased oscillatory neural activity of the basal ganglia and heightened synchronous activity across the basal ganglia thalamocortical networks in dopamine depleted states including PD (Costa et al., 2006; Gatev et al., 2006; Hammond et al., 2007; Eusebio et al., 2009). According to these reports, the pathological state of dopamine depletion results in increased synchronous oscillatory activity in the basal ganglia and its associated networks. Therapeutic measures such as dopaminergic medications and deep brain stimulation have been shown to reduce these oscillations and the associated increase in coherent neural activity across networks (Brown et al., 2001; Williams et al., 2002; Priori et al., 2004; Silberstein et al., 2005). The increase in cortico-cortical coupling seen in EEG data from PD patients has been shown to be associated with symptom severity (Silberstein et al., 2005). Additionally, decreases in corticocortical coupling due to L-DOPA and deep brain stimulation correlate with clinical improvement (Silberstein et al., 2005). The effect of dopaminergic modulation on coherence of oscillations in the basal ganglia thalamocortical networks has been shown in healthy individuals as well (Honey et al., 2003). Honey et al. (2003) assessed the changes in cortico-striatal network connectivity associated with different states of dopaminergic transmission caused by drug administration in healthy older adults. The authors observed increased functional connectivity between caudate and thalamus/ventral midbrain in decreased dopamine transmission states caused by Sulpiride, a dopamine D2 antagonist (Honey et al., 2003). 


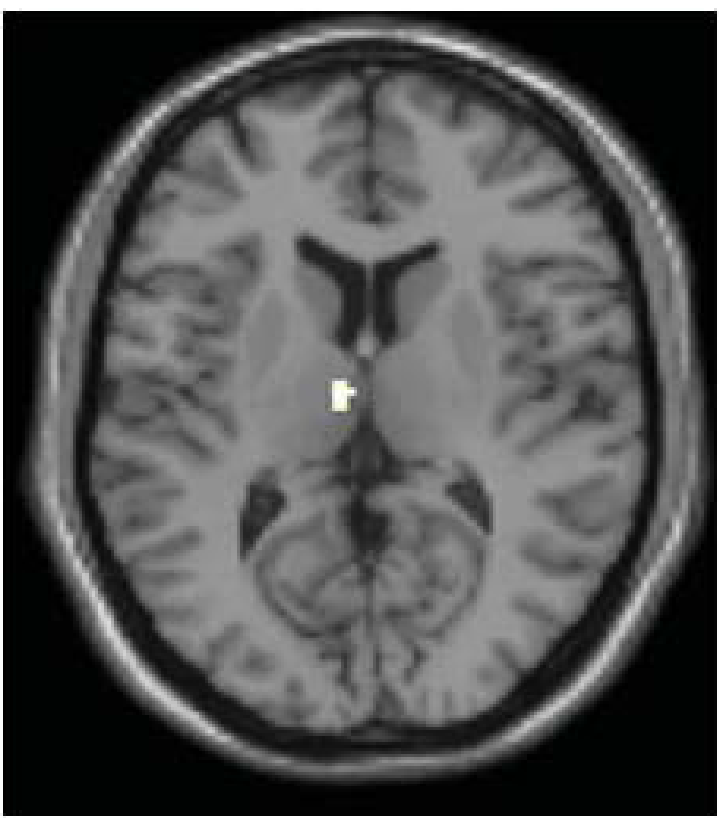

FIGURE 8 | Correlation between the difference in OFF and ON frequency content and OFF and ON connectivity in dorsal caudate. OFF-ON frequency content difference in $0.04-0.05 \mathrm{~Hz}$ of dorsal caudate was positively correlated with OFF-ON connectivity within the dorsomedial thalamus $(p<0.005$ uncorrected using the OFF-ON connectivity map as a mask).

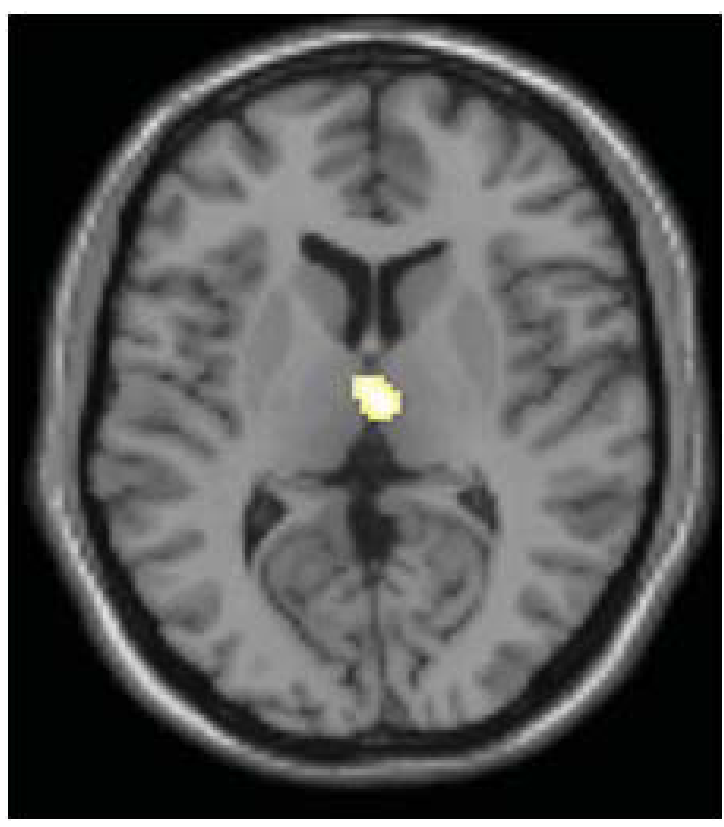

FIGURE 9 | Correlation between the difference in OFF and ON frequency content and OFF and ON connectivity in dorsal caudal putamen. OFF-ON frequency content difference in $0.03-0.04 \mathrm{~Hz}$ was positively correlated with OFF-ON connectivity differences within the dorsomedial thalamus $(p<0.005$, uncorrected using OFF-ON connectivity map as a mask).

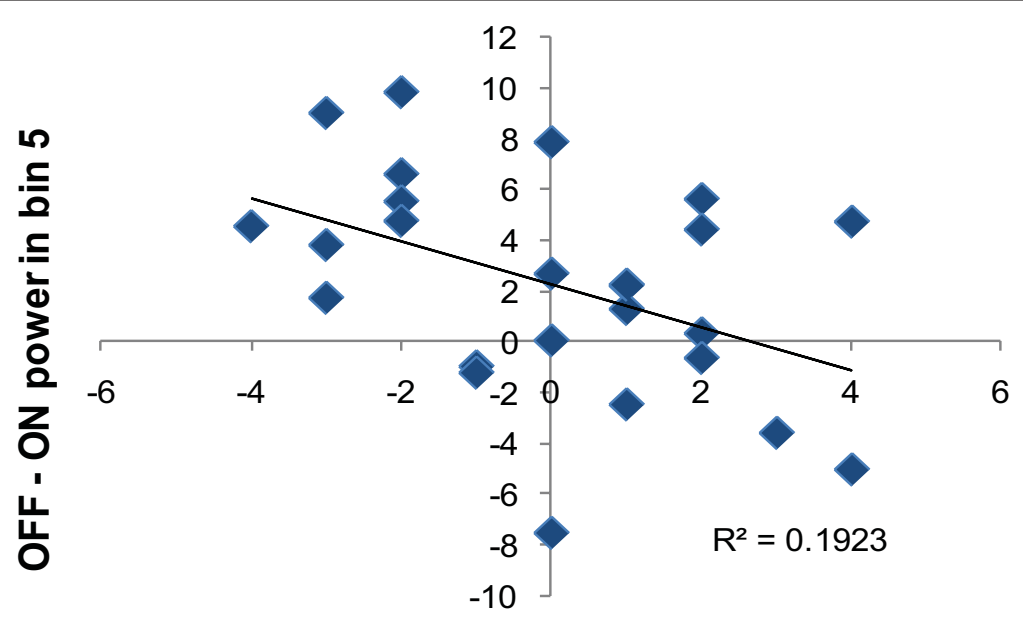

MOCA score OFF - ON

FIGURE 10 | Correlation between the difference in OFF and ON frequency content and OFF and ON MOCA performance difference. OFF-ON frequency power in $0.04-0.05 \mathrm{~Hz}$ of dorsal caudate (DC) negatively correlated with OFF-ON performance difference in MOCA $(r=0.44, p<0.05)$.

Oscillatory neural activity in PD and its modulation by drug and deep brain stimulation therapies have been examined by analyzing the frequency content of local field potential recordings or EEG/MEG data (Silberstein et al., 2003, 2005; Kuhn et al., 2004, 2006; Priori et al., 2004; Foffani et al., 2005; Weinberger et al., 2006; Stoffers et al., 2008). Some of these studies report that the PD-related increase in signal frequency content is most prominent in the beta frequency band (10-35 Hz) (Kuhn et al., 2004, 2006; Foffani et al., 2005; Weinberger et al., 2006). Others have found increased power in lower frequency bands including theta, alpha 1 and alpha 2 in PD (Stoffers et al., 2008). Studies evaluating the effect of dopaminergic therapy on modulation of brain signal frequency content have also 
shown that with medication, power decreases in the frequency range close to the beta band and concomitantly increases in the lower frequency band (2-7 Hz) (Silberstein et al., 2003; Priori et al., 2004).

In the current study, we compared the frequency content of the BOLD signal time course extracted from the striatal seed regions across medication states and between PD patients and controls. Due to the rate of fMRI data acquisition and BOLD signal preprocessing including low pass filtering, we were only able to look at the frequency range below $0.08 \mathrm{~Hz}$. However, given the sluggish nature of the hemodynamic response function (Haller and Bartsch, 2009), this should be sufficient to capture the frequency range of interest. We found an increase in power content of frequency bands in the range of $0.02-0.05 \mathrm{~Hz}$ and a decrease in power in the range of $<0.02 \mathrm{~Hz}$ for PD OFF compared to PD ON and controls. Furthermore for the comparison of PD OFF and PD ON, there were no significant interactions between medication and frequency bands for the inferior ventral striatum and the ventral rostral putamen. These two regions are the most ventral among the six seed locations, and are thus relatively intact in the early stages of PD (Bernheimer et al., 1973; Kish et al., 1988; Frey et al., 1996; Rakshi et al., 1999; Braak et al., 2003, 2006). Given this, our results suggest that L-DOPA affects a specific frequency range of resting state BOLD signal oscillations, and this effect is present in the more disease affected subregions of the striatum.

An exploratory analysis was performed to determine whether the L-DOPA-associated change in the resting state BOLD frequency content modulated the L-DOPA-associated connectivity differences in any brain regions. We used the frequency bands that showed a significant difference in signal content between PD ON and OFF. Our results showed that differences in signal content of the frequency band modulated the difference in connectivity strength between dorsal caudate and dorsomedial thalamus and also between dorsal caudal putamen and dorsomedial thalamus. That is, the more L-DOPA reduced power in the particular frequency band, the greater the reduction in connectivity between the striatal seed regions (i.e., dorsal caudate and dorsal caudal putamen) and dorsomedial thalamus. It is of note that the dorsomedial thalamus was commonly found in these analyses. Dorsomedial thalamus has traditionally been considered as the relay station for prefrontal and limbic connections and is involved in attention and alertness (Smythies, 1997). However, recent studies report the involvement of the dorsomedial thalamus in cortico-striatal circuitries (Cheatwood et al., 2003; Kunzle, 2006). Considering that our approach was exploratory, future studies are warranted to confirm these particular L-DOPA modulation effects in dorsomedial thalamus.

The L-DOPA-associated change in frequency content of the dorsal caudate signal was correlated with change in MOCA performance between ON and OFF L-DOPA. In other words, the more that L-DOPA reduced signal content in the frequency band the greater MOCA performance improved. To our knowledge, this is the first finding that shows L-DOPA associated shift in neural oscillation affects change in cognitive performance. Previously studies have shown that change in neural oscillation due to medication is correlated with motor improvement (Silberstein et al., 2005), however there has been no reports in terms of the relationship with cognitive improvements. Notably, the seed region we found this relationship with cognitive improvement in is the dorsal caudate, which predominantly has connections with the lateral prefrontal areas including the dorsolateral prefrontal cortex (Di Martino et al., 2008).

Our data showed some parallel findings to previous studies demonstrating changes in oscillatory neural activity in PD patients. Specifically we found changes in the low-frequency spontaneous fluctuations of the BOLD signal in PD patients at rest. A careful interpretation of our data is necessary however since the nature of BOLD signal oscillations could be different from oscillatory activity represented in local field potentials or EEG in previous studies (Silberstein et al., 2003, 2005; Kuhn et al., 2004, 2006; Priori et al., 2004; Foffani et al., 2005; Stoffers et al., 2008). The frequency range in our resting state BOLD signal was limited to below $0.08 \mathrm{~Hz}$ due to the nature of the neurovascular coupling (Haller and Bartsch, 2009) whereas the frequency range for local field potentials or EEG recordings is not limited to this low frequency range. Recently efforts have been made to understand the nature of the BOLD signal, in particular its relationship to neural activity. Simultaneous intracortical recordings and fMRI have demonstrated that the BOLD signal correlates with both local field potentials and multi-unit activity, but it is more accurately predicted by local field potentials (Logothetis et al., 2001; Goense and Logothetis, 2008). Simultaneous EEG and fMRI studies have also shown that patterns of fMRI activation can be explained by the frequency content of the EEG signal (Moosmann et al., 2003; Laufs, 2008; de Munck et al., 2009; Britz et al., 2010; Musso et al., 2010; Rosa et al., 2010). Additionally, studies have shown that the frequency content of the fMRI BOLD signal has behavioral relevance (Horovitz et al., 2008; Wu et al., 2008).

One interesting finding associated with the effect of L-DOPA in our patient group was that it seemed to overcorrect the hyperconnectivity of the cortico-striatal pathways as shown by our results comparing PD ON to controls. That is, there was an overall decrease in the level of cortico-striatal connectivity in PD ON compared to healthy controls. Irrespective of the striatal seed locations, there was a predominant decrease in connectivity between the striatum and the primary and secondary motor areas in PD ON, which was true even for seed regions that did not exhibit a relative hyperconnectivity for PD OFF relative to controls. Whether this hypoconnectivity for PD ON relative to controls is another side effect of L-DOPA that results in deleterious performance outcomes similar to the "dopamine-overdose effect" (see Cools, 2006 for review; Kwak et al., 2010) needs further investigation. The pattern of results may also be due to L-DOPA's selective effects on the more denervated striatal regions, typically the sensorimotor striatum and its motor cortical targets (Bernheimer et al., 1973; Kish et al., 1988; Rakshi et al., 1999; Braak et al., 2003, 2006).

As mentioned in the Results section, the resting state corticostriatal functional connectivity patterns in our data are in line with previous findings. That is, separable "cognitive" and "motor" circuitries were identified in healthy older adults and PD patients $\mathrm{ON}$ and OFF L-DOPA. However, one thing we did notice was that the cognitive and motor circuitries were less separable in our older adult group compared to what has been reported in previous studies conducted in healthy younger adults (Di Martino et al., 2008; Kelly et al., 2009). The reduced specificity of the cognitive and motor circuitries was even more apparent in our patient 
groups. One possible explanation for this discrepancy from previous findings is that the data processing and analysis streams were not identical to these previous studies, although we used the same MNI coordinates for the seed regions. Another possible explanation would be that the reduced specificity of the networks is due to age-related changes in neural recruitment considering that our participants were in their $60 \mathrm{~s}$ on average. Given this, the decrease in the exclusiveness of the cortico-striatal circuitries may be due to the age-related dedifferentiation of neural networks (Park and Reuter-Lorenz, 2009; Seidler et al., 2010). This topic awaits further investigation. With regards to $\mathrm{PD}$, a decrease in the relative separation of the cognitive and motor networks was even more apparent. That is, direct comparison of the caudate and putamen connectivity maps in the patient group demonstrated that the relative separation of the striatal networks present in controls was not observed in PD in either the ON or OFF L-DOPA state. This suggests that the cognitive and motor cortico-striatal networks become more diffuse and overlapping in PD in both the $\mathrm{ON}$ and OFF medication states.

We found that PD and L-DOPA were associated with shifts in the frequency content of resting state striatal signals, and this was correlated with connectivity strength and cognitive performance change, but not with changes in UPDRS scores. In fact the controlled dose of L-DOPA we used across all patients did not always improve UPDRS score, which suggests that it may have not been clinically efficient for some patients. Additionally, we cannot directly link the frequency range in resting state BOLD signal to the frequency

\section{REFERENCES}

Alexander, G. E., DeLong, M. R., and Strick, P. L. (1986). Parallel organization of functionally segregated circuits linking basal ganglia and cortex. Annu. Rev. Neurosci. 9, 357-381.

Bernheimer, H., Birkmayer, W., Hornykiewicz, O., Jellinger, K., and Seitelberger,F. (1973). Brain dopamine and the syndromes of Parkinson and Huntington. Clinical, morphological and neurochemical correlations. J. Neurol. Sci. 20, 415-455.

Biswal, B., Yetkin, F. Z., Haughton, V. M., and Hyde, J. S. (1995). Functional connectivity in the motor cortex of resting human brain using echoplanar MRI. Magn. Reson. Med. 34, 537-541.

Biswal,B.B.,Mennes, M.,Zuo,X.N.,Gohel, S., Kelly, C., Smith, S. M., Beckmann, C. F., Adelstein, J. S., Buckner, R. L., Colcombe, S., Dogonowski, A. M., Ernst, M., Fair, D., Hampson, M., Hoptman, M. J., Hyde, J.S., Kiviniemi, V. J., Kotter, R., Li, S. J., Lin, C. P., Lowe, M. J., Mackay, C., Madden, D. J., Madsen, K. H., Margulies, D. S., Mayberg, H. S., McMahon, K., Monk, C. S., Mostofsky, S. H., Nagel, B. J., Pekar, J. J., Peltier, S. J., Petersen, S. E., Riedl, V., Rombouts, S. A., Rypma, B., Schlaggar, B. L., Schmidt, S., Seidler, R. D., Siegle, G. J., Sorg,

ranges that were reported in EEG or local field potential studies. A further investigation combining EEG and resting state fMRI in $\mathrm{PD}$ patients will be required to address this issue.

In conclusion, our results showing a PD-associated increase in cortico-striatal functional connectivity and shifts in the power content of striatal signals parallel previous findings of increased coupled neural oscillatory activity in $\mathrm{PD}$, as measured with local field potentials and EEG. Moreover we found that the L-DOPA associated changes in BOLD signal oscillations modulate changes in connectivity and cognitive performance associated with L-DOPA. It is particularly interesting that our analysis of the low frequency fcMRI signal parallels previous EEG analyses of neural signal content at much higher frequencies. Considering that local field potential recordings can only be done in surgical settings and that EEG only captures cortical activity with low spatial resolution, whereas we found significant changes with fcMRI in mild to moderate stage patients, resting state fcMRI has great potential to be applied to further clinical research investigating the pathophysiology, progression, and treatment of PD.

\section{ACKNOWLEDGMENTS}

This study was supported by a grant from the Gustavus and Louise Pfeiffer Foundation (Rachael D. Seidler). We thank Darcy Huismann, Halley Feldman, Melissa Tan, Sarah Shin, Melanie Sottile, Mona Ramlawi, and Keith Newnham for assistance with data collection and analyses. We also thank J. Wayne Aldridge, Joshua Berke, and Bruno Averbeck for their insightful comments on an earlier draft of the manuscript.

tions between subthalamic nucleus and pallidum in Parkinson's disease. J. Neurosci. 21, 1033-1038.

Caballol, N., Marti, M. J., and Tolosa, E. (2007). Cognitive dysfunction and dementia in Parkinson disease. Mov. Disord. 22, S358-S366.

Cheatwood, J. L., Reep, R. L., and Corwin, J. V. (2003). The associative striatum: cortical and thalamic projections to the dorsocentral striatum in rats. Brain Res. 968, 1-14.

Cools, R. (2006). Dopaminergic modulation of cognitive function-implications for L-DOPA treatment in Parkinson's disease. Neurosci. Biobehav. Rev. 30, 1-23.

Costa, R. M., Lin, S. C., Sotnikova, T. D., Cyr, M., Gainetdinov, R. R., Caron, M. G., and Nicolelis, M. A. (2006). Rapid alterations in corticostriatal ensemble coordination during acute dopaminedependent motor dysfunction. Neuron 52, 359-369.

de Munck, J. C., Goncalves, S. I., Mammoliti, R., Heethaar, R. M., and Lopes da Silva, F. H. (2009). Interactions between different EEG frequency bands and their effect on alpha-fMRI correlations. Neuroimage 47, 69-76.

Di Martino, A., Scheres, A., Margulies, D. S., Kelly, A. M., Uddin, L. Q., Shehzad, Z., Biswal, B., Walters, J.
R., Castellanos, F. X., and Milham, M. P. (2008). Functional connectivity of human striatum: a resting state fMRI study. Cereb. Cortex 18, 2735-2747.

Eusebio, A., Pogosyan, A., Wang, S., Averbeck, B., Gaynor, L. D., Cantiniaux, S., Witjas, T., Limousin, P., Azulay, J. P., and Brown, P. (2009). Resonance in subthalamo-cortical circuits in Parkinson's disease. Brain 132, 2139-2150.

Fahn, S., Elton, R., and Committee., M.o.t.U.D. (1987). “The Unified Parkinson's Disease Rating Scale," in Recent Developments in Parkinson's Disease, Vol. 2, eds S. Fahn, C. D. Marsden, D. B. Calne, M. Goldstein (Florham Park, NJ: Macmillan Healthcare Information), 293-304.

Foffani, G., Bianchi, A. M., Baselli, G., and Priori, A. (2005). Movementrelated frequency modulation of beta oscillatory activity in the human subthalamic nucleus. J. Physiol. 568, 699-711.

Folstein, M.F., Folstein, S.E., and McHugh, P. R. (1975). “Mini-mental state”. A practical method for grading the cognitive state of patients for the clinician. J. Psychiatr. Res. 12, 189-198.

Fox, M. D., and Raichle, M. E. (2007). Spontaneous fluctuations in brain activity observed with functional 
magnetic resonance imaging. Nat. Rev. Neurosci. 8, 700-711.

Frey, K. A., Koeppe, R. A., Kilbourn, M. R., VanderBorght, T. M., Albin, R. L., Gilman, S., and Kuhl, D. E. (1996). Presynaptic monoaminergic vesicles in Parkinson's disease and normal aging. Ann. Neurol. 40, 873-884.

Gatev, P., Darbin, O., and Wichmann, T. (2006). Oscillations in the basal ganglia under normal conditions and in movement disorders. Mov. Disord. 21, 1566-1577.

Glover, G. H., and Law, C. S. (2001). Spiral-in/out BOLD fMRI for increased SNR and reduced susceptibility artifacts. Magn. Reson. Med. 46, 515-522.

Glover, G.H., Li, T. Q., and Ress, D. (2000). Image-based method for retrospective correction of physiological motion effects in AMRI: RETROICOR. Magn. Reson. Med. 44, 162-167.

Goense, J. B., and Logothetis, N. K. (2008). Neurophysiology of the BOLD fMRI signal in awake monkeys. Curr. Biol. 18, 631-640.

Goldman, R. I., Stern, J. M., Engel, J. Jr., and Cohen, M.S. (2002).Simultaneous EEG and fMRI of the alpha rhythm. Neuroreport 13, 2487-2492.

Greicius, M. (2008). Resting-state functional connectivity in neuropsychiatric disorders. Curr. Opin. Neurol. 21, 424-430.

Greicius, M. D., Kiviniemi, V., Tervonen, O., Vainionpaa, V., Alahuhta, S., Reiss, A. L., and Menon, V. (2008). Persistent default-mode network connectivity during light sedation. Hum. Brain Mapp. 29, 839-847.

Greicius, M. D., Supekar, K., Menon, V., and Dougherty, R. F. (2009). Restingstate functional connectivity reflects structural connectivity in the default mode network. Cereb. Cortex 19, 72-78.

Haber, S. N., and Knutson, B. (2010). The reward circuit: linking primate anatomy and human imaging. Neuropsychopharmacology 35, 4-26.

Haller, S., and Bartsch, A. J. (2009). Pitfalls in fMRI. Eur. Radiol. 19, 2689-2706.

Hammond, C., Bergman, H., and Brown, P. (2007). Pathological synchronization in Parkinson's disease: networks, models and treatments. Trends Neurosci. 30, 357-364.

Helmich, R. C., Derikx, L. C., Bakker, M., Scheeringa, R., Bloem, B. R., and Toni, I. (2010). Spatial remapping of cortico-striatal connectivity in Parkinson's disease. Cereb. Cortex 20, 1175-1186.

Hoehn, M. M., and Yahr, M. D. (1967). Parkinsonism: onset, progression and mortality. Neurology 17, 427-442.
Honey, G. D., Suckling, J., Zelaya, F., Long, C., Routledge, C., Jackson, S., Ng, V., Fletcher, P. C., Williams, S. C., Brown, J., and Bullmore, E. T. (2003). Dopaminergic drug effects on physiological connectivity in a human cortico-striato-thalamic system. Brain 126, 1767-1781.

Horovitz, S. G., Fukunaga, M., de Zwart, J. A., van Gelderen, P., Fulton, S. C., Balkin, T. J., and Duyn, J. H. (2008). Low frequency BOLD fluctuations during resting wakefulness and light sleep: a simultaneous EEG-fMRI study. Hum. Brain Mapp. 29, 671-682.

Huynh, H., and Feldt, L. S. (1970). Conditions under which the mean square ratios in repeated measures designs have exact F-distributions. J. Am. Stat. Assoc. 65, 1582-1589.

Jenkinson, M., Bannister, P., Brady, M., and Smith, S. (2002). Improved optimization for the robust and accurate linear registration and motion correction of brain images. Neuroimage 17, 825-841.

Kelly, C., de Zubicaray, G., Di Martino, A., Copland, D. A., Reiss, P. T., Klein, D. F., Castellanos, F. X., Milham, M. P., and McMahon, K. (2009). L-DOPA modulates functional connectivity in striatal cognitive and motor networks: a double-blind placebo-controlled study. J. Neurosci. 29, 7364-7378.

Kish, S. J., Shannak, K., and Hornykiewicz, O. (1988). Uneven pattern of dopamine loss in the striatum of patients with idiopathic Parkinsons disease: pathophysiologic and clinical implications. N. Engl. J. Med. 318, 876-880.

Kuhn, A. A., Kupsch, A., Schneider, G. H., and Brown, P. (2006). Reduction in subthalamic $8-35 \mathrm{~Hz}$ oscillatory activity correlates with clinical improvement in Parkinson's disease. Eur. J. Neurosci. 23, 1956-1960.

Kuhn, A. A., Williams, D., Kupsch, A., Limousin, P., Hariz, M., Schneider, G. H., Yarrow, K., and Brown, P. (2004). Event-related beta desynchronization in human subthalamic nucleus correlates with motor performance. Brain 127, 735-746.

Kunzle, H. (2006). Thalamo-striatal projections in the hedgehog tenrec. Brain Res. 1100, 78-92.

Kwak, Y., Muller, M. L., Bohnen, N. I., Dayalu, P., Seidler, R. D. (2010). Effect of dopaminergic medications on the time course of explicit motor sequence learning in Parkinson's disease. J. Neurophysiol. 103, 942-949.

Laufs, H. (2008). Endogenous brain oscillations and related networks detected by surface EEG-combined fMRI. Hum. Brain Mapp. 29, 762-769.

Laufs, H., Kleinschmidt, A., Beyerle, A., Eger, E., Salek-Haddadi, A., Preibisch,
C., and Krakow, K. (2003). EEGcorrelated fMRI of human alpha activity. Neuroimage 19, 1463-1476.

Levy, R., Ashby, P., Hutchison, W. D., Lang, A. E., Lozano, A. M., and Dostrovsky, J. O. (2002). Dependence of subthalamic nucleus oscillations on movement and dopamine in Parkinson's disease. Brain 125, 1196-1209.

Logothetis, N. K., Pauls, J., Augath, M., Trinath, T., and Oeltermann, A. (2001). Neurophysiological investigation of the basis of the fMRI signal. Nature 412, 150-157.

Monk, C. S., Peltier, S. J., Wiggins, J. L., Weng, S. J., Carrasco, M., Risi, S. and Lord, C. (2009). Abnormalities of intrinsic functional connectivity in autism spectrum disorders. Neuroimage 47, 764-772.

Moosmann, M., Ritter, P., Krastel, I., Brink, A., Thees, S., Blankenburg, F., Taskin, B., Obrig, H., and Villringer, A. (2003). Correlates of alpha rhythm in functional magnetic resonance imaging and near infrared spectroscopy. Neuroimage 20, 145-158.

Musso, F., Brinkmeyer, J., Mobascher, A., Warbrick, T., and Winterer, G. (2010). Spontaneous brain activity and EEG microstates. A novel EEG/ fMRI analysis approach to explore resting-state networks. Neuroimage $52,1149-1161$.

Nasreddine,Z.S., Phillips, N.A., Bedirian, V. Charbonneau, S., Whitehead, V., Collin, I., Cummings, J. L., and Chertkow, H. (2005). The Montreal Cognitive Assessment, MoCA: a brief screening tool for mild cognitive impairment. J. Am. Geriatr. Soc. 53, 695-699.

Nichols, T., and Hayasaka, S. (2003). Controlling the familywise error rate in functional neuroimaging: a comparative review. Stat. Methods Med. Res. 12, 419-446.

Oppenheim, A., Schafer, R., and Buck, J. (1999). Discrete-Time Signal Processing. Upper Saddle River, NJ: Prentice Hall.

Park, D. C., and Reuter-Lorenz, P. (2009). The adaptive brain: aging and neurocognitive scaffolding. Annu. Rev. Psychol. 60, 173-196.

Peltier, S. J., LaConte, S. M., Niyazov, D. M., Liu, J.Z., Sahgal, V., Yue, G. H., and $\mathrm{Hu}$, X. P. (2005). Reductions in interhemispheric motor cortex functional connectivity after muscle fatigue. Brain Res. 1057, 10-16.

Peltier, S. J., Polk, T. A., and Noll, D. C. (2003). Detecting low-frequency functional connectivity in fMRI using a self-organizing map (SOM) algorithm. Hum. Brain Mapp. 20, 220-226.

Priori, A., Foffani, G., Pesenti, A., Tamma, F., Bianchi, A. M., Pellegrini,
M., Locatelli, M., Moxon, K. A., and Villani, R. M. (2004). Rhythm-specific pharmacological modulation of subthalamic activity in Parkinson's disease. Exp. Neurol. 189, 369-379.

Rakshi, J. S., Uema, T., Ito, K., Bailey, D. L. Morrish, P. K., Ashburner, J., Dagher, A., Jenkins, I. H., Friston, K. J., and Brooks, D. J. (1999). Frontal, midbrain and striatal dopaminergic function in early and advanced Parkinson's disease-A 3D [F-18]dopa-PET study. Brain 122, 1637-1650.

Rogers, B. P., Morgan, V. L., Newton, A. T., and Gore, J. C. (2007). Assessing functional connectivity in the human brain by fMRI. Magn. Reson. Imaging 25, 1347-1357.

Rosa,M.J., Kilner,J., Blankenburg, F.,Josephs, O., and Penny,W. (2010). Estimating the transfer function from neuronal activity to BOLD using simultaneous EEGfMRI. Neuroimage 49, 1496-1509.

Seidler, R. D., Bernard, J. A., Burutolu, T. B., Fling, B. W., Gordon, M. T., Gwin, J. T., Kwak, Y., and Lipps, D. B. (2010). Motor control and aging: links to agerelated brain structural, functional, and biochemical effects. Neurosci. Biobehav. Rev. 34, 721-733.

Shohamy, D., Myers, C. E., Geghman, K. D., Sage, J., and Gluck, M. A. (2006). L-DOPA impairs learning, but spares generalization, in Parkinson's disease. Neuropsychologia 44, 774-784.

Silberstein, P., Kuhn, A. A., Kupsch, A., Trottenberg, T., Krauss, J. K., Wohrle, J. C., Mazzone, P., Insola, A., Di Lazzaro, V., Oliviero, A., Aziz, T., and Brown, P. (2003). Patterning of globus pallidus local field potentials differs between Parkinson's disease and dystonia. Brain 126, 2597-2608.

Silberstein, P., Pogosyan, A., Kuhn, A. A., Hotton, G., Tisch, S., Kupsch, A., Dowsey-Limousin, P., Hariz, M. I., and Brown, P. (2005). Cortico-cortical coupling in Parkinson's disease and its modulation by therapy. Brain 128 , 1277-1291.

Smythies, J. (1997). The functional neuroanatomy of awareness: with a focus on the role of various anatomical systems in the control of intermodal attention. Conscious. Cogn. 6, 455-481.

Stam, C. J. (2010). Use of magnetoencephalography (MEG) to study functional brain networks in neurodegenerative disorders. J. Neurol. Sci. 289, 128-134.

Stoffers, D., Bosboom, J. L., Deijen, J. B., Wolters, E., Stam, C. J., and Berendse, H.W.(2008). Increased cortico-cortical functional connectivity in early-stage Parkinson's disease: an MEG study. Neuroimage 41, 212-222.

van Meer, M. P., van der Marel, K., Wang, K., Otte, W.M.,El Bouazati, S., Roeling, 
T. A., Viergever, M. A., Berkelbach van der Sprenkel, J. W., and Dijkhuizen, R. M. (2010). Recovery of sensorimotor function after experimental stroke correlates with restoration of resting-state interhemispheric functional connectivity. J. Neurosci. 30, 3964-3972.

Vercammen, A., Knegtering, H., Liemburg, E. J., Boer, J.A., and Aleman, A. (2010). Functional connectivity of the temporo-parietal region in schizophrenia: effects of rTMS treatment of auditory hallucinations. J. Psychiatr. Res. 44, 725-731.

Vincent, J. L., Patel, G. H., Fox, M. D., Snyder, A. Z., Baker, J. T., Van Essen, D. C., Zempel, J. M., Snyder, L. H.,
Corbetta, M., and Raichle, M. E. (2007). Intrinsic functional architecture in the anaesthetized monkey brain. Nature 447, 83-86.

Weinberger, M., Mahant, N., Hutchison, W.D., Lozano, A. M., Moro, E., Hodaie, M., Lang, A. E., and Dostrovsky, J. O. (2006). Beta oscillatory activity in the subthalamic nucleus and its relation to dopaminergic response in Parkinson's disease. J. Neurophysiol. 96, 3248-3256.

Williams, D., Tijssen, M., Van Bruggen, G., Bosch, A., Insola, A., Di Lazzaro, V., Mazzone, P., Oliviero, A., Quartarone, A., Speelman, H., and Brown, P. (2002). Dopamine-dependent changes in the functional connectivity between basal ganglia and cerebral cortex in humans. Brain 125, 1558-1569.

Wu, C.W., Gu, H., Lu, H.,Stein, E.A.,Chen, J. H., and Yang, Y. (2008). Frequency specificity of functional connectivity in brain networks. Neuroimage 42 1047-1055.

Wu, T., Wang, L., Chen, Y., Zhao, C., Li, K., and Chan, P. (2009). Changes of functional connectivity of the motor network in the resting state in Parkinson's disease. Neurosci. Lett. 460, 6-10.

Conflict of Interest Statement: The authors declare that the research was conducted in the absence of any commercial or financial relationships that could be construed as a potential conflict of interest.
Received: 13 May 2010; paper pending published: 04 June 2010; accepted: 23 August 2010; published online: 15 September 2010.

Citation: Kwak Y, Peltier S, Bohnen NI, Müller MLTM, Dayalu P and Seidler RD (2010) Altered resting state cortico-striatal connectivity in mild to moderate stage Parkinson's disease. Front. Syst. Neurosci. 4:143 doi:10.3389/fnsys.2010.00143 Copyright $\odot 2010$ Kwak, Peltier, Bohnen, Müller, Dayalu and Seidler.This is an open-access article subject to an exclusive license agreement between the authors and the Frontiers Research Foundation, which permits unrestricted use, distribution, and reproduction in any medium, provided the original authors and source are credited. 


\section{SUPPLEMENTARY MATERIAL}

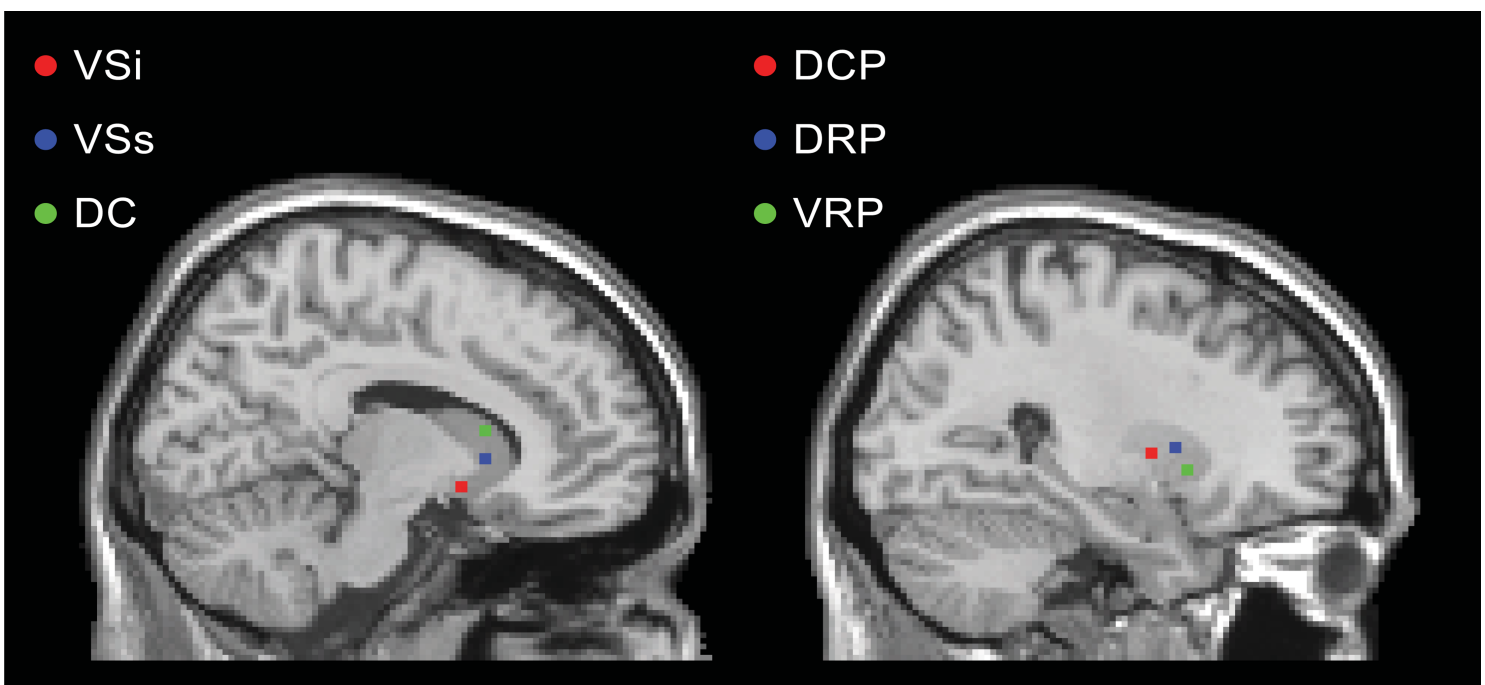

FIGURE S1 | Location of the six striatal seed regions. The left shows ( $x=11)$ the location of the three caudate seeds: inferior ventral striatum (VSi), superior ventral striatum (VSs), and dorsal caudate (DC). The right $(x=28)$ shows the locations of the three putamen seeds: dorsal caudal putamen (DCP), dorsal rostral putamen (DRP), and ventral rostral putamen (VRP).

Table S1 | Demographic and clinical variables of PD patients.

\begin{tabular}{|c|c|c|c|c|c|c|c|}
\hline & Age & Gender & $\begin{array}{l}\text { Disease } \\
\text { duration (years) }\end{array}$ & $\begin{array}{l}\text { Affected } \\
\text { body side }\end{array}$ & $H$ and $Y$ & UPDRS_ON & UPDRS_OFF \\
\hline PD_01 & 62 & $M$ & 6 & $\mathrm{R}$ & 2.5 & 15 & 16 \\
\hline PD_02 & 70 & $M$ & 8 & $L$ & 2 & 23 & 19 \\
\hline PD_03 & 70 & $\mathrm{M}$ & 7 & $\mathrm{R}$ & 2 & 14 & 21 \\
\hline PD_04 & 52 & $M$ & 6 & $\mathrm{R}$ & 2.5 & 16 & 20 \\
\hline PD_05 & 71 & $M$ & 13 & $\mathrm{R}$ & 2.5 & 18 & 24 \\
\hline PD_06 & 69 & $M$ & 12 & $\mathrm{R}$ & 2 & 20 & 24 \\
\hline PD_07 & 60 & $M$ & 3 & $\mathrm{R}$ & 1.5 & 10 & 9 \\
\hline PD_08 & 65 & $M$ & 8 & $\mathrm{R}$ & 2.5 & 21 & 27 \\
\hline PD_09 & 63 & $\mathrm{M}$ & 7 & $\mathrm{~L}$ & 2.5 & 28 & 28 \\
\hline PD_10 & 69 & $M$ & 9 & $\mathrm{R}$ & 2 & 24 & 23 \\
\hline PD_11 & 66 & $M$ & 1 & $\mathrm{~L}$ & 2 & 9 & 9 \\
\hline PD_12 & 67 & $\mathrm{M}$ & 9 & $\mathrm{R}$ & 2.5 & 14 & 14 \\
\hline PD_13 & 73 & $\mathrm{M}$ & 2 & $\mathrm{R}$ & 2.5 & 18 & 14 \\
\hline PD_14 & 51 & $\mathrm{M}$ & 2 & $\mathrm{R}$ & 2 & 9 & 8 \\
\hline PD_15 & 68 & $M$ & 2 & $\mathrm{~L}$ & 2 & 25 & 27 \\
\hline PD_16 & 72 & $M$ & 4 & $\mathrm{~L}$ & 2.5 & 27 & 26 \\
\hline PD_17 & 59 & $\mathrm{~F}$ & 2 & $\mathrm{R}$ & 2 & 5 & 6 \\
\hline PD_18 & 64 & $M$ & 7 & $\mathrm{R}$ & 2 & 8 & 6 \\
\hline PD_19 & 64 & $M$ & 3 & $L$ & 2 & 28 & 30 \\
\hline PD_20 & 80 & $M$ & 6 & $\mathrm{R}$ & 2 & 11 & 13 \\
\hline PD_21 & 50 & $M$ & 3 & $\mathrm{R}$ & 1.5 & 13 & 13 \\
\hline PD_22 & 62 & $\mathrm{M}$ & 2 & $\mathrm{R}$ & 2 & 10 & 17 \\
\hline PD_23 & 51 & $M$ & 3 & $\mathrm{R}$ & 2.5 & 32 & 37 \\
\hline PD_24 & 56 & $\mathrm{~F}$ & 1 & $L$ & 2 & 11 & 21 \\
\hline PD_25 & 55 & $\mathrm{~F}$ & 3 & $\mathrm{R}$ & 2 & 15 & 14 \\
\hline Mean & 63.6 & & 5.2 & & 2.1 & 17.0 & 18.6 \\
\hline S.D. & 7.8 & & 3.4 & & 0.3 & 7.4 & 8.1 \\
\hline
\end{tabular}


Table S2 | MNI coordinates of the local maxima of brain regions showing functional connectivity with the six striatal seed regions in controls.

\begin{tabular}{|c|c|c|c|c|c|c|}
\hline \multirow[t]{2}{*}{ Seed } & \multirow[t]{2}{*}{ Region } & \multirow[t]{2}{*}{ BA } & \multicolumn{3}{|c|}{ MNI coordinates } & \multirow[t]{2}{*}{$T$-value } \\
\hline & & & $x$ & $y$ & $z$ & \\
\hline Inferior ventral striatum & Superior temporal gyrus & 42 & 56 & 16 & 8 & 10.2 \\
\hline \multirow[t]{4}{*}{ Superior ventral striatum } & Lingual gyrus & 18 & -4 & 72 & -4 & 13.59 \\
\hline & Superior parietal lobule & 7 & 26 & 62 & 54 & 12.58 \\
\hline & Middle frontal gyrus & 6 & 30 & -2 & 56 & 11.89 \\
\hline & Paracentral gyrus & 6 & -6 & 28 & 76 & 10.82 \\
\hline \multirow[t]{3}{*}{ Dorsal caudate } & Supramarginal gyrus & 40 & 48 & 50 & 30 & 13.65 \\
\hline & Middle frontal gyrus & 46 & 54 & 28 & 22 & 11.45 \\
\hline & Middle frontal gyrus & 10 & 32 & 54 & 18 & 11.34 \\
\hline \multirow[t]{3}{*}{ Dorsal caudal putamen } & Anterior cingulate gyrus & 32 & 6 & 14 & 36 & 10.54 \\
\hline & Postcentral gyrus & 7 & 14 & 54 & 50 & 10.39 \\
\hline & Precentral gyrus & 4 & 34 & 18 & 58 & 10.21 \\
\hline \multirow[t]{7}{*}{ Dorsal rostral putamen } & Insula & 13 & 38 & 8 & 6 & 13.49 \\
\hline & Superior frontal gyrus & 9 & 36 & 46 & 30 & 12.63 \\
\hline & Superior parietal lobule & 7 & 10 & 68 & 56 & 12.52 \\
\hline & Inferior parietal lobule & 40 & 62 & 30 & 22 & 12.25 \\
\hline & Middle temporal gyrus & 19 & 46 & 74 & 18 & 11.05 \\
\hline & Inferior frontal gyrus & 44 & 52 & 4 & 22 & 10.16 \\
\hline & Cerebellum lobule V & & 12 & 56 & 26 & 12.12 \\
\hline Ventral rostral putamen & Superior temporal gyrus & 21 & 58 & 24 & 12 & 17.79 \\
\hline
\end{tabular}




\section{Cognitive and motor networks in PD OFF and PD ON}

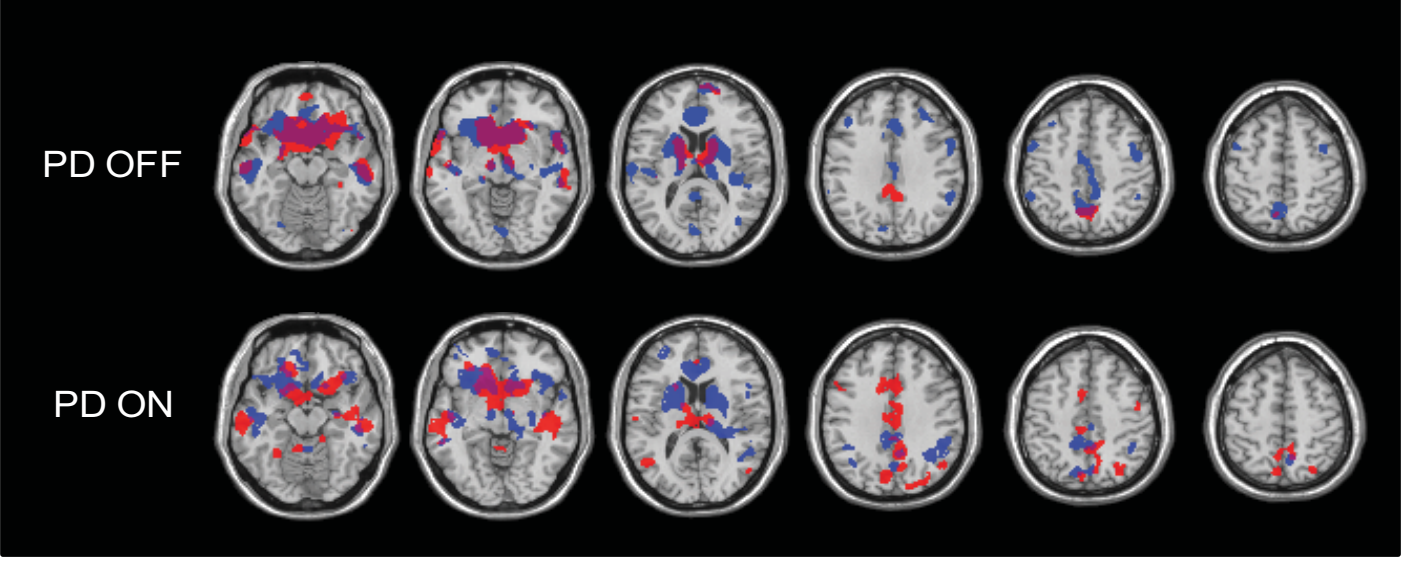

FIGURE S2 | Cognitive (seed: inferior ventral striatum, red) and motor (seed: dorsal rostral putamen, blue) networks in PD OFF (top) and PD ON (bottom). $z=-15,-5,15,35,45,55$. FWE-corrected $p<0.05$. 
Table S3 | MNI coordinates of the local maxima of brain regions showing functional connectivity with the six striatal seed regions in PD OFF.

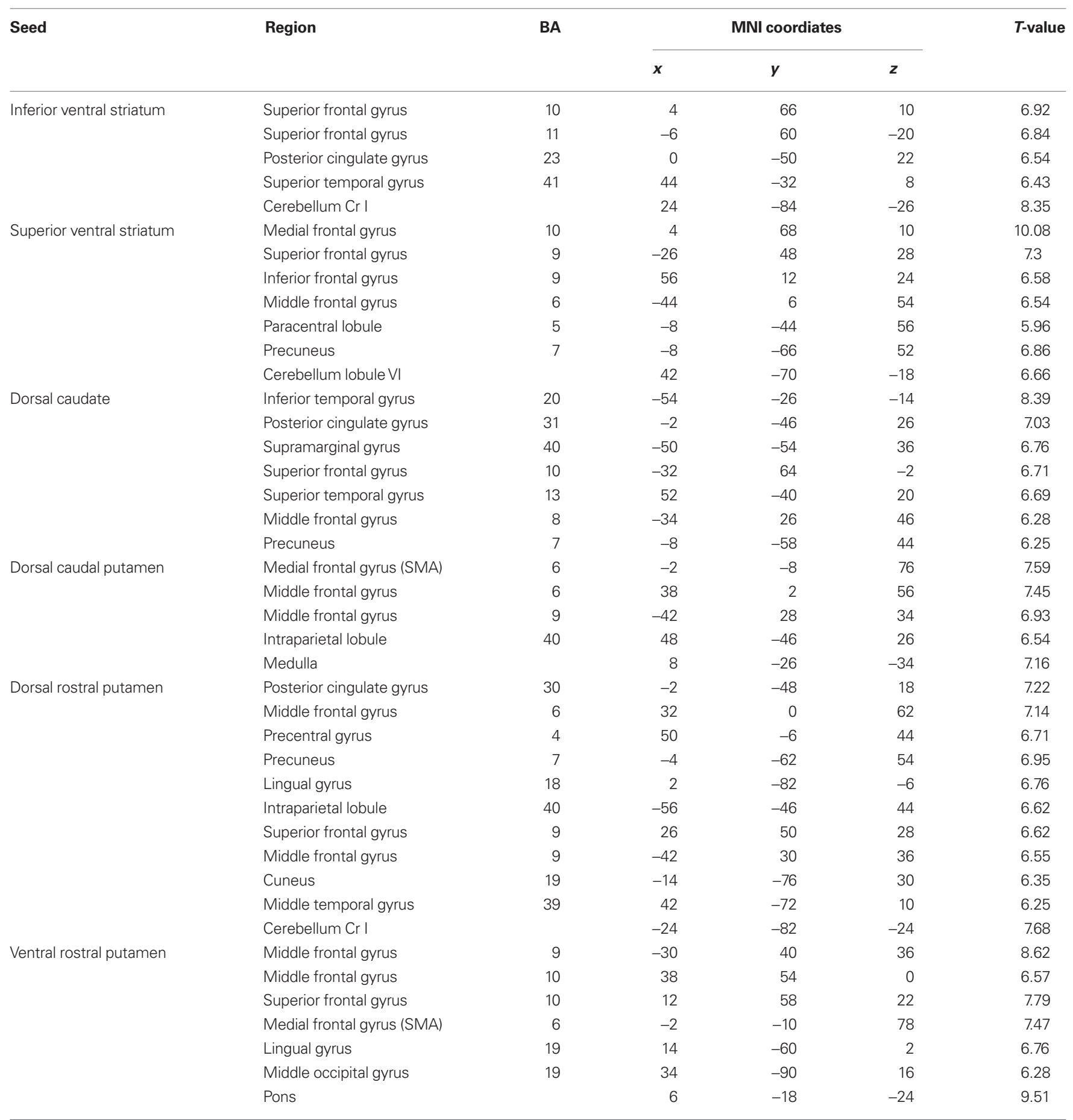


Table S4 | MNI coordinates of the local maxima of brain regions showing functional connectivity with the six striatal seed regions in PD ON.

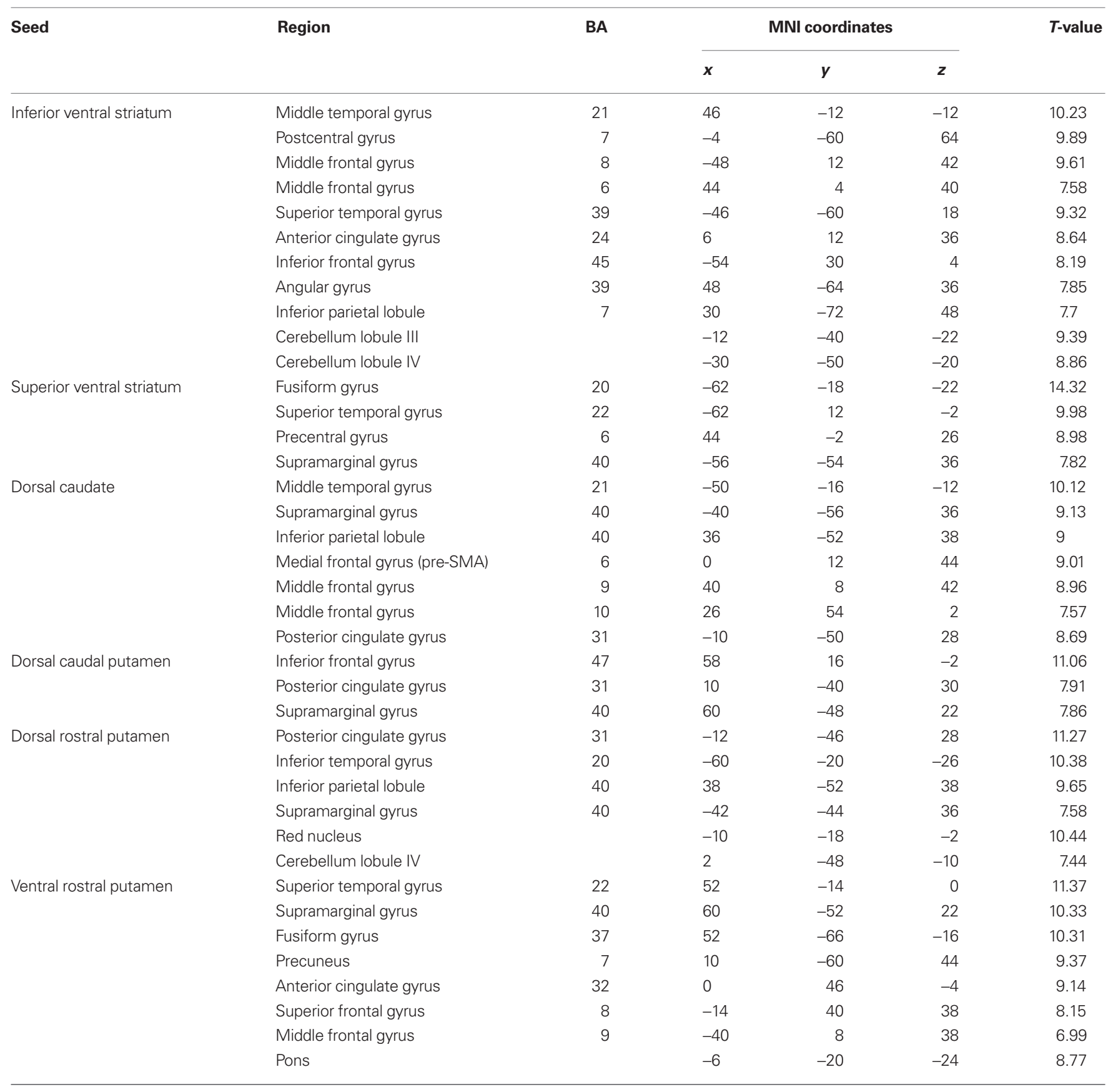


Table S5 | MNI coordinates of the local maxima of brain regions showing greater functional connectivity in PD OFF than controls.

\begin{tabular}{|c|c|c|c|c|c|c|}
\hline \multirow[t]{2}{*}{ Seed } & \multirow[t]{2}{*}{ Region } & \multirow[t]{2}{*}{ BA } & \multicolumn{3}{|c|}{ MNI coordinates } & \multirow[t]{2}{*}{$T$-value } \\
\hline & & & $x$ & $y$ & $z$ & \\
\hline \multirow[t]{3}{*}{ Dorsal caudal putamen } & Inferior temporal gyrus & 20 & 48 & -10 & -20 & 4.47 \\
\hline & Inferior temporal gyrus & 21 & -58 & -16 & -16 & 3.73 \\
\hline & Middle temporal gyrus & 21 & 54 & -18 & -8 & 3.52 \\
\hline \multirow[t]{6}{*}{ Dorsal rostral putamen } & Rectal gyrus & 11 & 12 & 30 & -18 & 4.42 \\
\hline & Middle temporal gyrus & 21 & 50 & -6 & -24 & 3.93 \\
\hline & Inferior temporal gyrus & 20 & -46 & -8 & -32 & 3.81 \\
\hline & Anterior cingulate gyrus & 32 & -18 & 34 & -8 & 3.62 \\
\hline & Orbital frontal gyrus & 47 & -38 & 26 & -22 & 3.93 \\
\hline & Medial frontal gyrus & 10 & 10 & 60 & 8 & 3.43 \\
\hline
\end{tabular}


Table S6 | MNI coordinates of the local maxima of brain regions showing greater functional connectivity in controls than PD ON.

\begin{tabular}{|c|c|c|c|c|c|c|}
\hline \multirow[t]{2}{*}{ Seed } & \multirow[t]{2}{*}{ Region } & \multirow[t]{2}{*}{ BA } & \multicolumn{3}{|c|}{ MNI coordinates } & \multirow[t]{2}{*}{$T$-value } \\
\hline & & & $x$ & $y$ & $z$ & \\
\hline & Superior frontal gyrus & 11 & -24 & 52 & -12 & 3.87 \\
\hline & Superior frontal gyrus & 10 & -22 & 66 & -8 & 3.82 \\
\hline & Medial frontal gyrus (SMA) & 6 & -6 & -22 & 68 & 3.73 \\
\hline & Cerebellum $\mathrm{Cr}$ II & & -18 & -82 & -40 & 4.63 \\
\hline \multirow[t]{10}{*}{ Superior ventral striatum } & Postcentral gyrus & 3 & 38 & -30 & 64 & 5.25 \\
\hline & Precentral gyrus & 4 & -30 & -16 & 68 & 4.93 \\
\hline & Superior frontal gyrus & 6 & -26 & 2 & 64 & 3.54 \\
\hline & Medial frontal gyrus (SMA) & 6 & -6 & -22 & 72 & 4.07 \\
\hline & Middle frontal gyrus & 6 & -32 & 10 & 50 & 3.7 \\
\hline & Inferior frontal gyrus & 46 & 48 & 48 & 4 & 4.23 \\
\hline & Superior parietal lobule & 7 & 18 & -74 & 54 & 3.88 \\
\hline & Cuneus & 18 & -18 & -90 & 26 & 3.68 \\
\hline & Lingual gyrus & 17 & 6 & -92 & -4 & 3.85 \\
\hline & Globus pallidus & & -18 & -8 & -6 & 3.78 \\
\hline \multirow[t]{6}{*}{ Dorsal caudate } & Postcentral gyrus & 3 & 38 & -30 & 70 & 5.99 \\
\hline & Precentral gyrus & 4 & -30 & -16 & 70 & 5.68 \\
\hline & Precentral gyrus & 6 & 32 & -10 & 68 & 3.99 \\
\hline & Middle frontal gyrus & 9 & 42 & 42 & 30 & 5.17 \\
\hline & Middle frontal gyrus & 10 & -36 & 62 & 18 & 4.11 \\
\hline & Inferior frontal gyrus & 46 & 48 & 46 & 4 & 4.07 \\
\hline \multirow{11}{*}{ Dorsal caudal putamen } & Precentral gyrus & 6 & 22 & -16 & 74 & 4.23 \\
\hline & Medial frontal gyrus (pre-SMA) & 6 & -6 & 14 & 52 & 3.97 \\
\hline & Medial frontal gyrus & 6 & 18 & -16 & 50 & 3.84 \\
\hline & Medial frontal gyrus & 32 & -10 & 28 & 34 & 4.06 \\
\hline & Middle frontal gyrus & 8 & -30 & 34 & 40 & 3.96 \\
\hline & Middle frontal gyrus & 9 & 36 & 12 & 34 & 3.46 \\
\hline & Superior frontal gyrus & 10 & -32 & 54 & 26 & 3.71 \\
\hline & Superior parietal lobule & 7 & -42 & -54 & 62 & 4.17 \\
\hline & Lingual gyrus & 17 & -4 & -92 & -4 & 3.67 \\
\hline & Postcentral gyrus & 2 & -40 & -78 & -2 & 3.46 \\
\hline & Putamen & & -18 & -10 & 0 & 3.87 \\
\hline \multirow[t]{8}{*}{ Dorsal rostral putamen } & Precentral gyrus & 4 & -58 & -20 & 40 & 3.57 \\
\hline & Paracentral lobule & 5 & 16 & -36 & 48 & 3.47 \\
\hline & Postcentral gyrus & 2 & -68 & -24 & 34 & 3.76 \\
\hline & Superior frontal gyrus & 6 & -36 & -8 & 70 & 7.28 \\
\hline & Superior frontal gyrus & 10 & -36 & 56 & 26 & 4.68 \\
\hline & Superior frontal gyrus & 8 & -20 & 48 & 50 & 4.4 \\
\hline & Middle frontal gyrus & 8 & 46 & 22 & 46 & 4.06 \\
\hline & Medial frontal gyrus & 32 & -10 & 24 & 36 & 3.7 \\
\hline
\end{tabular}


Table S6 | Continued

\begin{tabular}{|c|c|c|c|c|c|c|}
\hline \multirow[t]{2}{*}{ Seed } & \multirow[t]{2}{*}{ Region } & \multirow[t]{2}{*}{ BA } & \multicolumn{3}{|c|}{ MNI coordinates } & \multirow[t]{2}{*}{$T$-value } \\
\hline & & & $x$ & $y$ & $z$ & \\
\hline & Middle frontal gyrus & 10 & 34 & 58 & 22 & 3.56 \\
\hline & Inferior frontal gyrus & 44 & -44 & 16 & 12 & 4.23 \\
\hline & Superior parietal lobule & 7 & -18 & -82 & 52 & 4.41 \\
\hline & Middle temporal gyrus & 39 & -36 & -70 & 24 & 3.7 \\
\hline & Middle occipital gyrus & 19 & -40 & -74 & 8 & 4.01 \\
\hline & Lingual gyrus & 18 & -4 & -92 & -4 & 3.91 \\
\hline & Precuneus & 7 & -4 & -62 & 68 & 3.67 \\
\hline \multirow[t]{9}{*}{ Ventral rostral putamen } & Precentral gyrus & 4 & -36 & -26 & 70 & 4.9 \\
\hline & Precentral gyrus & 6 & -38 & 16 & 8 & 4.39 \\
\hline & Superior parietal lobule & 5 & 16 & -36 & 52 & 4.66 \\
\hline & Lingual gyrus & 18 & 18 & -92 & -6 & 4.45 \\
\hline & Cuneus & 17 & -32 & -78 & 10 & 3.89 \\
\hline & Fusiform gyrus & 37 & -48 & -48 & -10 & 4.06 \\
\hline & Transverse temporal gyrus & 41 & 52 & -16 & 12 & 3.85 \\
\hline & Cerebellum lobule VIIIA & & 22 & -72 & -48 & 4.42 \\
\hline & Cerebellum Cr II & & -8 & -78 & -42 & 3.87 \\
\hline
\end{tabular}


Table S7 | Brain regions showing greater functional connectivity in PD OFF than PD ON.

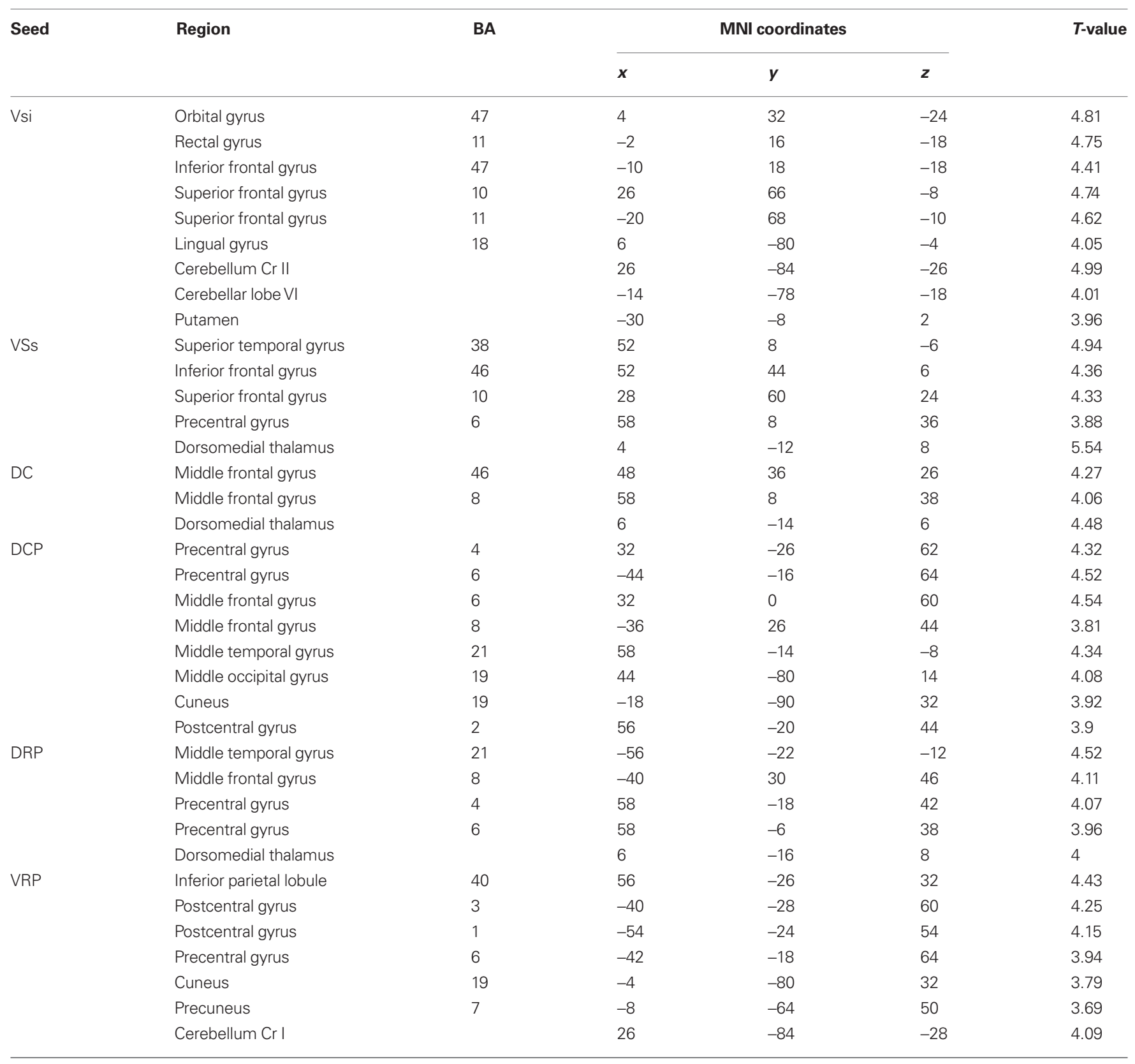



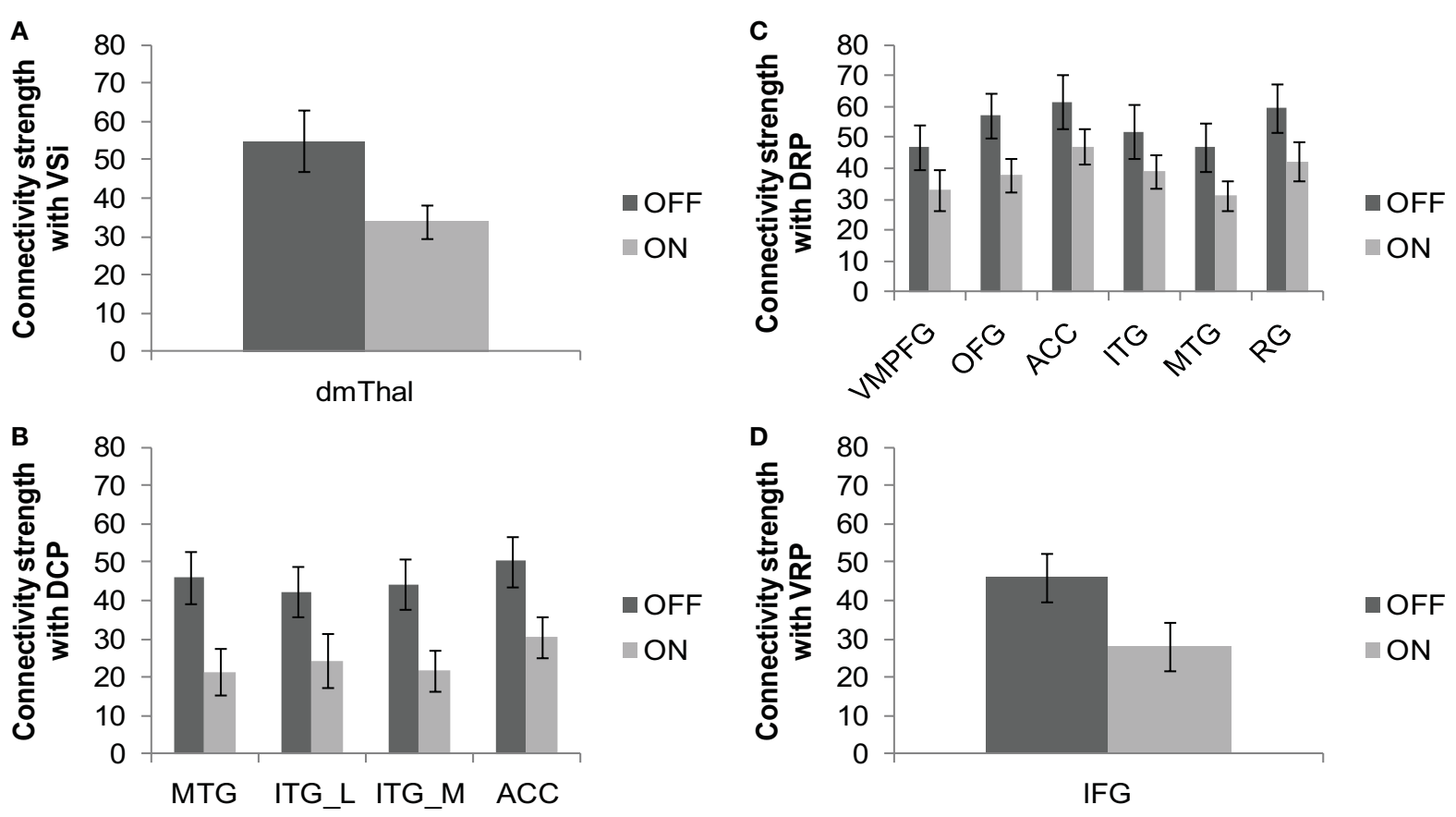

FIGURE S3 | Connectivity strength difference between PD OFF and PD ON in ROls defined in PD OFF versus controls comparison. Connectivity strength between VSi (A), VSs (B), DRP (C), and VRP (D) and each of the ROIs are compared across PD OFF and PD ON. VSi, inferior ventral striatum; dmThal, dorsomedial thalamus; DCP, dorsal caudal putamen; MTG, middle temporal gyrus; ITG_L, inferior temporal gyrus in the less affected side; ITG_R, inferior temporal gyrus in the more affected side; ACC, anterior

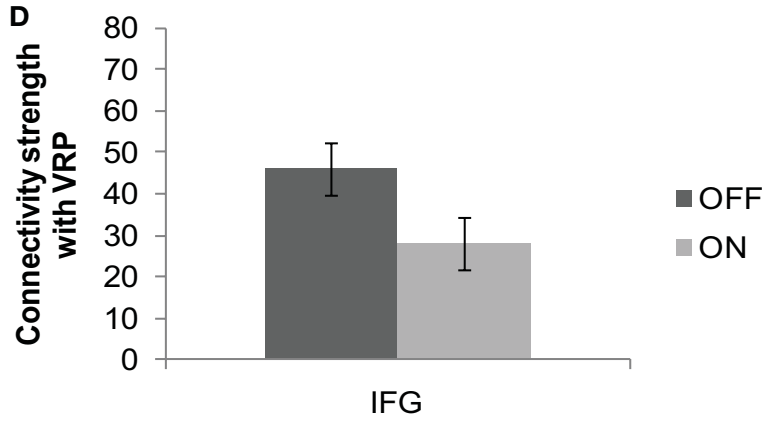

cingulated cortex; DRP; dorsal rostral putamen; VMPFC, ventromedial prefrontal gyrus; OFG, orbital frontal gyrus; RG, rectal gyrus; VRP, ventral rostral putamen; inferior frontal gyrus. Error bars indicate standard error. For all comparisons we found significant main effect of medication status showing decrease in strength of connectivity in PD ON compared to PD OFF with the exception of DRP and VRP which showed marginally significant medication main effects. 